\title{
Particles and Connections on Four-Manifolds
}

\author{
J.C. Hurtubise 1
}

\begin{abstract}
For the simple, simply connected classical gauge groups $G$, we build spaces $\mathcal{P}(X, Y)$ homotopy equivalent to the spaces $\mathcal{B}(X, Y)$ of gauge equivalence classes of pairs ( $G$-connections on a fourmanifold $X$, trivialisations of the $G$-bundle over $Y \subset X)$. The space consists of configurations of particle-like connections, each corresponding to an instanton over $S^{4}$.
\end{abstract}

\section{Introduction.}

In understanding the homotopy type of an infinite dimensional space, it can be very useful to have some model for the space which expresses it as a limit of finite dimensional subspaces. An outstanding example of this is the May-Milgram model [May, Mi] for $\Omega^{n} \Sigma^{n}(X)$, the $n$-th fold loop space of the $n$-th fold suspension of a space $X$, which is described as an infinite particle limit of the spaces $J_{k}(X)$ of configurations of $k$ unordered $X$-labelled points in the $n$-plane, i.e., each point is labelled by an element of $X$. ( For $n=1$, this model is due to James [J].) This model carries in a natural way all the homology operations that one can perform on the space $\Omega^{n} \Sigma^{n}(X)$, essentially by moving points around, and this allows a quite complete description of the homology of the loop space [CLM]. In this paper our object of study will be the spaces $\mathcal{B}(X)$ of gauge equivalence classes of based connections on a principal $G$-bundle over a compact four-manifold $X$ and our aim will be to build a particle model for $\mathcal{B}(X)$, in the spirit of the May-Milgram model, so that again one has natural operations on homology. Also, the space $\mathcal{B}(X)$ has on it a natural energy functional, the Yang-Mills functional, and the relative topology of $\mathcal{B}(X)$ and the (finite-dimensional) moduli space $\mathcal{M}(X)$ of minima of this functional (instantons) has been an essential tool over the last ten years in understanding the differential topology of four-manifolds.

\footnotetext{
${ }^{1}$ During the preparation of this work the author was supported by grants from NSERC and FCAR.
} 
Our particle model ties in well with the Yang-Mills functional, in that the particle-like connections used will have almost minimal energy.

The spaces $\mathcal{B}(X)$ have associated to them a natural charge, or degree $k$, which is simply the first Pontrjagin class of the principal bundle. A conjecture due to Atiyah and Jones [AJ], stated originally for the foursphere, states that the inclusion $\mathcal{M}_{k}(X) \rightarrow \mathcal{B}_{k}(X)$ induces a homotopy equivalence through a range which increases with $k$. As the $\mathcal{B}_{k}(X)$ are homotopy equivalent, the Atiyah-Jones conjecture would allow us to define a homotopy model for $\mathcal{B}_{k}(X)$ as a suitable limit of the spaces $\mathcal{M}_{k}(X)$. Over a large open set of $\mathcal{M}_{k}$, the connections exhibit particle-like behaviour in that they are concentrated in small open neighbourhoods of a finite set of points, so that in the $\mathcal{M}_{k}$, or in a suitable limit, one would have something approximating a May-Milgram type particle model.

The conjecture has not been proven for a general compact four-manifold, though Taubes has proven a weaker version of it in [T2]. We will exploit instead the fact that the conjecture has been verified for the four-sphere [BHMM, Ti1, Ti2, Ki], and that the conformal invariance of the YangMills functional allows us to restrict ourselves to a subset of $\mathcal{M}_{k}\left(S^{4}\right)$ of connections whose curvature is essentially concentrated in a small ball. One can then graft these connections into an arbitrary four-manifold, and obtain a family of particle-like connections in $\mathcal{B}(X)$ which are almost minima of the Yang-Mills functional for that manifold. It is this family which will turn out to be homotopy equivalent to $\mathcal{B}(X)$. Taubes has given in [T3] another model for $\mathcal{B}(X)$; his construction has the virtue of exploiting the Morse theory of the Yang-Mills functional, giving a description which eventually could generalise to other functionals. It is, however, considerably more complex, in that it involves not only the minima but the other critical points of the Yang-Mills functional, and also requires a "multi-scale construction" for the glueing in of the $S^{4}$ instantons. Similar techniques allow him in [T2] to show that the limit of the $\mathcal{M}_{k}(X)$ is weakly homotopy equivalent to $\mathcal{B}(X)$. For finite $k$, the space is, however, not easily tractable.

The version we propose is not directly tied to the Yang-Mills functional on $X$, and exploits the fact that the Atiyah-Jones conjecture holds for $S^{4}$. In some sense, the multi-scale construction of Taubes is subsumed in the moduli space of $S^{4}$. Also, the finite-level spaces in our construction are better understood, and indeed, given a plausible analytic assumption can be seen to exhibit the type of homotopy stability that one desires. The particle nature of the connections used also makes them more amenable to the techniques of homotopy theory, for example to performing Dyer-Lashof type operations. 
Our model does provides a space, homotopy equivalent to $\mathcal{B}(X)$, of connections in $\mathcal{B}(X)$ of almost minimal energy, and so can still be viewed as a first step in proving the Atiyah-Jones conjecture. The work of Taubes indicates to us how families of connections of almost minimal energy on a four-manifold can be deformed into the space of true minima. In essence, there is an obstruction in a finite dimensional space which must vanish for the deformation to take place. A similar situation occurs when considering when an arbitrary configuration of poles and principal parts on a Riemann surface corresponds to a meromorphic map: one obtains an obstruction in the first cohomology group of the structure sheaf. In [Hu], it was shown how quite generally, one could arrange for the latter obstruction to be deformed to zero over a family of sufficiently high codimension, allowing a retraction on the level of homotopy groups; it is quite conceivable that such a theorem could be seen to hold here, giving a homotopy equivalence through a range between $\mathcal{B}(X)$ and a subspace of $\mathcal{M}(X)$ consisting of concentrated instantons. In turn, one could hope for an equivalence through a range between these concentrated instantons and the full instanton moduli space, as a sort of distant cousin of the Lefschetz hyperplane theorem for algebraic varieties [Li].

It will be convenient to work with more general spaces $\mathcal{B}(X, Y)$ of pairs

$$
\text { (connections on } X, \text { trivialisations on } Y \text { ), }
$$

where $Y$ is a suitably nice open set of $X$, and $X$ is not necessarily compact. We give some of the basic properties of the $\mathcal{B}(X, Y)$ in section two. Our "particles" that we use to build the model of $\mathcal{B}(X, Y)$ are not localised at points, but rather on small open sets. This turns out to be quite a major headache, and section three is devoted to developing the requisite tools for dealing with it. In section four, we build the homotopy model $\mathcal{P}\left(S^{4}, D^{4}\right)$ for $\mathcal{B}\left(S^{4}, D^{4}\right)$. This is essentially the instanton moduli space, but where the connections are modified to be flat outside of disks, so that the operations of glueing together two connections are easier to perform. In section 5, we transfer this model to an arbitrary pair $(X, Y)$, building $\mathcal{P}(X, Y)$, and proving that it does have the desired homotopy type. Section 6 considers more directly the finite charge spaces $\mathcal{P}_{k}(X, Y)$. We conclude in section 7 with a few comments.

The author would like to thank C. H. Taubes for clarifying some delicate technical questions. 


\section{Spaces of based connections.}

Let $X$ be a four dimensional smooth manifold with or without boundary, and let $Y$ be an open subset of $X$ containing the ends and the boundary of $X$, so that $X \backslash Y$ is compact. We will suppose that $X \backslash Y$ and $Y$ are both non empty and of finite type, so that in particular they both have a finite number of components. We will also suppose that $X \backslash Y$ is an 4-manifold with boundary smoothly embedded in $X$, so that there is a "collar" of $Y$, giving a family $Y_{t}, t \in[-1,1]$ of open neighbourhoods with $Y_{t}$ relatively compact in $Y_{t^{\prime}}$ for $t<t^{\prime}, Y_{0}=Y$, and with $Y_{t}, t>-1$ deforming diffeomorphically to $Y_{t^{\prime}}$ by diffeomorphisms which are the identity on $Y_{(-1+t) / 2}$. We will call such pairs $(X, Y)$ "admissible". (We will occasionally allow $X \backslash Y$ to have corners. This causes no difficulty). We also introduce the following notation: if $X$ has a boundary $\partial X$, then one can thicken $\partial X$ to a collar $\hat{\partial} X$ so that $(X, \hat{\partial} X)$ is admissible.

Let $G$ be a simple, simply connected compact classical Lie group, that is either $S U(n), \operatorname{Spin}(n)$ or $S p(n)$ : these are the groups for which the AtiyahJones conjecture over $S^{4}$ is known. Let us fix over $X$ a sequence $E_{k}$ of principal $G$-bundles with $p_{1}\left(E_{k}, T_{k}\right)=k \in H^{4}(X, Y ; \mathbb{Z})$. Along with trivialisations $T_{k}$ of $E_{k}$ over $\bar{Y}$. We will say that another trivialisation $T^{\prime}$ of $E_{k}$ over $\bar{Y}$ is admissible if $T_{k}$ and $T^{\prime}$ are related by a map $\bar{Y} \rightarrow G$ which is homotopic to the constant map.

Let $A_{k}(X, Y)$ be the space of pairs

( $G$-connections on $E_{k}$ over $X$, admissible trivialisation of $E_{k}$ over $\bar{Y}$ ).

Let $G_{k}(X, Y)$ be the group of gauge transformations of $E_{k}$ over $X$ which are homotopic to the identity over $\bar{Y}$, and set

$$
\mathcal{B}_{k}(X, Y)=A_{k}(X, Y) / G_{k}(X, Y)
$$

The space $\mathcal{B}_{k}(X, Y)$ has, up to homotopy, other interpretations. First, let $G_{k}(X)$ be the group of all gauge transformations of $E_{k}$ over $X$. We will say that a trivialisation $T^{\prime}$ of $E_{k}$ over $\bar{Y}$ is permissible if it lies in the $G_{k}(X)$-orbit of $T_{k}$. Alternately, as we have trivialisations $T_{k}$ of our bundles $E_{k}$ over $Y$, the gauge group $G_{k}(X)$ give after restriction to $\bar{Y}$ elements of $\operatorname{Map}(\bar{Y}, G)$, while those in $G_{k}(X, Y)$ give elements of $\operatorname{Map}^{0}(\bar{Y}, G)$, the connected component of the trivial map. We say that $f \in \operatorname{Map}(\bar{Y}, G)$ extends to $X$ if it lies in the image of $G_{k}(X, Y)$. In fact, all of $\operatorname{Map}^{0}(\bar{Y}, G)$ extends to $X$; more generally the maps which extend are a union of connected components of $\operatorname{Map}(\bar{Y}, G)$ : 
Lemma (2.2). If $f: \bar{Y} \rightarrow G$ is homotopic to the constant map, then $f$ extends to $X$.

Proof. Let $F_{t}$ be the homotopy of $f$ to the constant map with value the identity. One can use the restriction of $F_{t}$ to the boundary of $Y$ to extend $f$ to the collar of $Y$ in such a way that the extension is constant on the boundary of $Y_{1}$, after which $f$ extends trivially to the rest of $X$ as the trivial gauge transformation.

We note that this shows that an admissible trivialisation is permissible.

Let $a_{k}(X, Y)$ be the space of pairs

(G-connections on $E_{k}$ over $X$, permissible trivialisation of $E_{k}$ over $\left.\bar{Y}\right)$.

Then,

$$
\mathcal{B}_{k}(X, Y)=a_{k}(X, Y) / G_{k}(X) .
$$

We have simply augmented both the space of trivialisations and the gauge transformations. Next, let $\hat{A}_{k}(X, Y)$ be the space of connections on $E_{k}$ which vanish in the $T_{k}$-trivialisation over $\bar{Y}$, and $\hat{G}_{k}(X, Y)$ the gauge transformations which are trivial over $Y$.

Proposition (2.4). $\mathcal{B}_{k}(X, Y)$ is homotopic to

$$
\hat{\mathcal{B}}_{k}(X, Y)=\hat{A}_{k}(X, Y) / \hat{G}_{k}(X, Y) .
$$

Proof. We choose once and for all lifts $\tilde{\rho}_{t, t^{\prime}}$ of the retractions $\rho_{t, t^{\prime}}: Y_{t} \rightarrow Y_{t^{\prime}}$, $t^{\prime}<t$ to $E_{k}$ which preserve the trivialisations $T_{k}$. The map $\tilde{\rho}_{1,0}$ gives an "extension" of any trivialisation $T$ over $\bar{Y}$ to a $T_{1}$ over $Y_{1}$, in such a way that $T_{1}=T$ over $Y_{-\frac{1}{2}}$, say. For any such $T$, let $\alpha_{0}$ be the connection over $Y_{1}$ corresponding to $T_{1}$, i.e., $\alpha_{0}=0$ in the $T_{1}$-trivialisation. We then choose a smooth function

$$
\varphi: X \times[0,1] \rightarrow[0,1]
$$

with $\varphi(X \times\{0\})=1, \varphi((X \backslash Y) \times[0,1])=1$ and $\varphi\left(\left(Y_{-\frac{1}{2}}\right) \times\{1\}\right)=0$. We then set, for all $(\alpha, T)$ in $A_{k}(X, Y)$ :

$$
R(\alpha, T)=\left(\varphi \alpha+(1-\varphi) \alpha_{0}, T\right)
$$


This defines an equivariant retraction of $A_{k}(X, Y)$ to $A_{k}^{F}(X, Y)$, a subspace of $A_{k}(X, Y)$ consisting of pairs $\left(\alpha, T^{\prime}\right)$, where $\alpha$ is flat on $Y_{-\frac{1}{2}}$ and trivial ("zero") there with respect to the $T$-trivialisation. We note that if $\alpha$ is zero in the $T$-trivialisation, then $g \cdot \alpha$ is zero in the $g \cdot T$ trivialisation. One has

$$
A_{k}(X, Y) / G_{k}(X, Y) \simeq A_{k}^{F}(X, Y) / G_{k}(X, Y) .
$$

One then uses the fact that $G_{k}(X, Y)$ acts transitively on the admissible trivialisations to reduce to the space

$$
\hat{A}_{k}^{F}(X, Y)=\left\{(\alpha, T) \in A_{k}^{F}(X, Y) \mid T=T_{k}\right\},
$$

and so:

$$
A_{k}^{F}(X, Y) / G_{k}(X, Y) \simeq \hat{A}_{k}^{F}(X, Y) / \hat{G}_{k}(X, Y)
$$
sions

Finally, $\hat{A}_{k}^{F}(X, Y)$ is not quite the same as $\hat{A}_{k}(X, Y)$, but one has inclu-

$$
\hat{A}_{k}(X, Y) \subset \hat{A}_{k}^{F}(X, Y) \subset \hat{A}_{k}\left(X, Y_{-\frac{1}{2}}\right) \subset \tilde{\rho}_{0,-\frac{1}{2}}\left(\hat{A}_{k}^{F}(X, Y)\right)
$$

defining equivariant homotopy equivalences.

Proposition (2.8). Let $Y^{\prime} \subset Y, Y^{\prime} \neq \Phi$ be nice open sets, and let the trivialisations $T_{k}(Y), T_{k}\left(Y^{\prime}\right)$ be compatible. The natural map

$$
\mathcal{B}_{k}(X, Y) \longrightarrow \mathcal{B}_{k}\left(X, Y^{\prime}\right)
$$

is a fibration with fiber

$$
\operatorname{Map}_{X}\left(\left(Y, Y^{\prime}\right),(G, e)\right)
$$

the subspace of $\operatorname{Map}\left(\left(Y, Y^{\prime}\right),(G, e)\right)$ consisting of maps which extend to $X$.

Proof. We have that

$$
\mathcal{B}_{k}(X, Y)=a_{k}(x, Y) / G(X) \quad \text { and } \quad \mathcal{B}_{k}\left(X, Y^{\prime}\right)=a_{k}\left(X, Y^{\prime}\right) / G(X) .
$$

The fiber of the restriction map $a_{k}(X, Y) \rightarrow a_{k}\left(X, Y^{\prime}\right)$ is, however, $\operatorname{Map}_{X}\left(\left(Y, Y^{\prime}\right),(G, e)\right)$.

We emphasize that the trivialisations $T_{k}$ allow us to define for each component of $X \backslash Y$ a relative Pontrjagin class, so that the total Pontrjagin class of the pair $\left(E_{k}, T_{k}\right)$ is given by a multi-index $k=\left(k_{1}, \ldots, k_{s}\right)$. The existence of several components is the reason for defining admissible trivialisations, as, in flattening a connection with respect to a trivialisation, one wants to prevent "charge" from "leaking" from one component to another. 
Lemma (2.10). For all $\kappa$, the spaces $\mathcal{B}_{k}(X, Y)$ are homotopy equivalent and are connected.

Proof. The homotopy equivalences are given by the well known loop sum or glueing constructions, as in [DK]. The connectedness follows from that of $A_{\kappa}(X, Y)$ and the space of admissible trivialisations.

When it is not necessary to specify $k$, we will simply let the multi-index be implicit, and refer to the spaces as $\mathcal{B}(X, Y)$. It is however useful to bear these components in mind, for example when restricting from $\mathcal{B}(X, Y)$ to $\mathcal{B}\left(X, Y^{\prime}\right)$ for $Y^{\prime} \subset Y$. The inclusion $X \backslash Y \subset X \backslash Y^{\prime}$ typically amalgamates certain connected components of $X \backslash Y$, while also allowing for new ones in $X \backslash Y$. The "charges" $k=\left(k_{1}, \ldots, k_{s}\right)$ get summed and rearranged accordingly.

Lemma (2.11). Let $X=X_{1} \cup X_{2}, Y=Y_{1} \cup Y_{2}$ with $X_{1} \cap X_{2}=Y_{1} \cap Y_{2}$. Suppose that the trivialisations on $Y_{1}, Y_{2}$ are compatible. Then

$$
\mathcal{B}(X, Y) \simeq \mathcal{B}\left(X_{1}, Y_{1}\right) \times \mathcal{B}\left(X_{2}, Y_{2}\right) .
$$

Lemma (2.12) (Excision). Let $V$ be closed in $Y$. Then

$$
\mathcal{B}(X, Y)=\mathcal{B}(X \backslash V, Y \backslash V) .
$$

The proofs are straightforward. As a particular case, we can take $V$ to be the closure of $Y_{0}$. This gives us

$$
\mathcal{B}(X, Y)=\mathcal{B}(X \backslash Y, \hat{\partial}(X \backslash Y))
$$

and so, from (2.11), we can consider each component of $X \backslash Y$ separately. From now on, we take the multi-index $k$ to be an integer.

\section{Particles and disks.}

\section{3.a. Amalgamations.}

We will be building a homotopy-theoretic "particle model" for the space of connections on a bundle, in analogy with the May-Milgram model for $\Omega^{n} \Sigma^{n} X$. We will use for this spaces of concentrated "particle like" connections, which are flat outside small disks. This makes them more amenable 
to the type of "loop sum" additions of configurations that one would like to perform.

The "particles" we will use are thus localized on disks, not at points. The size of these disks can be an obstruction to moving them around as points; we get around this by building a rescaling into the model to allow them to be as small as need be. Another problem is that when the disks come together or coalesce, their localisation is not always well defined. We will give in the next few paragraphs a few notions and lemmas which will. be of use in getting around this problem. Constructions similar in spirit to what follows can be found in $[\mathrm{KM}]$. We fix a metric on $X$, with bounded curvature.

Let $A$ be a finite set, with some measure on $\mu$ on it of total mass 1 . Let $B_{\rho}$ be a geodesically convex ball in $X$, and, if $\Delta$ is a positive bound for the sectional curvatures on $X$, let us ask that $4 \rho<\pi \Delta^{-1 / 2}$. Following Karcher $[\mathrm{Ka}]$, we define a center of mass for a map $f: A \rightarrow X$. To do this, consider the function

$$
P_{f}(m)=\int_{A} d(m, f(a))^{2} d a
$$

for $m \in \bar{B}_{\rho}$. One has $[\mathrm{Ka}]$ :

Theorem (3.2). With the above assumptions, the function $P_{f}$ is convex, with a unique interior minimum $C_{f}$ in $B_{\rho}$, the centre of mass of $f$. The map $(f, \mu) \rightarrow C_{f}$ is continuous, and uniform in $f$.

This centre of mass using the counting measure will allows us to think of $n$ points which are close as one big point localised at the centre of mass. As points vary in $X$, we want to think of them as distinct when they are far apart, and agglomerated when they are close. This is done as follows.

Let $S P^{n}(X)$ be the $n$-th symmetric product of $X$. We note that if $X$ has $r$ connected components, then the components of $S P^{n}(X)$ are labelled by $r$-tuples of integers $\left(k_{1}, k_{2}, \ldots, k_{r}\right), k_{i} \geq 0, \sum k_{i}=n$. We will write elements $\xi$ of $S P^{n}(X)$ as sums $\sum a_{i} p_{i}, a_{i} \in \mathbb{Z}^{+}, p_{i}$ distinct $\in X$. The partitions $\left\{V_{i}\right\}_{i \in I}$ of a set have a natural ordering, given by "clustering": set $\left\{V_{i}\right\} \leq\left\{V_{i}^{\prime}\right\}$ if each element $V_{i}^{\prime}$ of the second partition can be written as a union of sets $V_{i}$ in the first partition. There is a similar ordering on the partitions of an integer. The space $S P^{n}(X)$ admits a stratification corresponding to this order, given by multiplicity pattern, i.e. the integers $a_{i}$ in the sum $\sum a_{i} p_{i}$. If $K:\left\{a_{1} \geq a_{2} \geq \cdots a_{j}\right\}$ is a partition of $n$, let $S_{K}$ be the corresponding 
stratum of $S P^{n}(X)$. One has

$$
\bar{S}_{K}=\bigcup_{K \leq K^{\prime}} S_{K^{\prime}}
$$

Definition. Let $\xi=\sum a_{i} p_{i}$ be an element of $S P^{n}(X)$. An amalgamation of $\xi$ is a partition of $\xi$ into disjoint $\zeta_{j} \in S P^{n_{j}}(X), \sum_{j} n_{j}=n$, so that $\xi=\sum_{j} \zeta_{j}$. Here disjoint means that each $p_{i}$ appears in only one $\zeta_{j}$. The word amalgamation is used to indicate that each $\zeta_{j}$ is to be thought of as one composite particle, agglomerating the $p_{i}$ that compose it.

There is a natural ordering on amalgamations, corresponding to the ordering of partitions. We also remark that there is a natural length function on amalgamations, given simply by the number of clusters $\zeta_{j}$. Passing to a larger amalgamation always decreases this length. The minimal amalgamation of $\xi=\sum_{i=1}^{s} a_{i} p_{i}$ has length $s$; its maximal amalgamation always has length 1 .

Definition. Let $d=(d(1), d(2), d(3), \ldots)$ be an increasing sequence of positive real numbers and $\varepsilon>0$ a positive real number. Let $\xi=\sum a_{i} p_{i}$ be an element of $S P^{n}(X)$. A $(d, \varepsilon)$ amalgamation of $\xi$ is a partition of $\xi$ into disjoint $\zeta_{j} \in S P^{n_{j}}(X), \sum_{j} n_{j}=n$, such that each $p_{i}$ in $\zeta_{j}$ is within $\varepsilon d\left(n_{j}\right)$ of the centre of gravity of the points in $\zeta_{j}$.

Lemma (3.4). There exists a sequence $d=d(1), d(2), d(3), \ldots$ such that for all $\varepsilon_{0}(n)>\varepsilon>0$, any $X \in S P^{n}(X)$ has a unique maximal $(d, \varepsilon)$ amalgamation, and if $\left\{\zeta_{j}\right\}, \zeta_{j} \in S P^{n_{j}}(X)$ is this amalgamation, then for $j \neq i$, the centres of gravity $\bar{\zeta}_{j}, \bar{\zeta}_{i}$ of $\zeta_{j}, \zeta_{i}$ respectively are separated by at least $3 \varepsilon\left(d\left(n_{j}\right)+d\left(n_{i}\right)\right)$.

Proof. The $\varepsilon$ factor is an overall scaling which for the purposes of the proof can chosen arbitrarily, as long as it is sufficiently small; for the proof, we will just set it to one. We define $d(i)$ inductively, starting with $d(1)=1$. Let $\xi_{1} \in S P^{n_{1}}(X), \xi_{2} \in S P^{n_{2}}(X)$ with all the points $p_{i, j}$ of $\xi_{i}$ within $d\left(n_{i}\right)$ of their centres of gravity $\bar{\xi}_{i}$. For $\xi_{1}$ and $\xi_{2}$ to be united in a larger amalgamation if the $\bar{\xi}_{i}$ are less than $3\left(d\left(n_{1}\right)+d\left(n_{2}\right)\right)$ apart , one simply has to choose $d\left(n_{1}+n_{2}\right)$ large enough so that all points of $\xi_{1}$ and $\xi_{2}$ lie within $d\left(n_{1}+n_{2}\right)$ of their common centre of gravity. This is ensured for example by

$$
d(m)>C \frac{(4 n) d(n)+(m+4 n) d(m-n)}{m} .
$$


for all $0<n<m$, allowing an inductive definition. (The constant $C$ would be one if the manifold were flat; it can be taken greater than one to allow for curvature.)

Let us fix such a $d$, and fix a "window" $[0, a]$, with $a$ sufficiently small for the centres of mass to be well defined for points in a ball of radius $a d(n)$. We then define for each

$$
\xi=\sum_{i=1}^{s(\xi)} a_{i} p_{i} \in S P^{n}(X)
$$

its amalgamation sequence $\left(\varepsilon_{i}, \zeta^{i}\right), i=s(\xi)-1, \ldots, 1$ where $0<\varepsilon_{s(\xi)-1} \leq$ $\cdots \leq \varepsilon_{r(\xi)+1}<\varepsilon_{r(\xi)}=\cdots=\varepsilon_{1}=a, \zeta^{i}=\left\{\zeta_{j}^{i}\right\}, \zeta_{j}^{i} \in S P^{n_{j}^{i}}(X), \sum n_{j}^{i}=n$. One considers, for each $\varepsilon>0$, the maximal $(d, \varepsilon)$ amalgamation. As a function of $\varepsilon$, this is locally constant, except at a finite number of exceptional values $\rho_{\mu}$, where it changes from $\zeta^{\mu}$ to a bigger $\zeta^{\mu^{\prime}}$ as one increases $\varepsilon$. Let $\varepsilon_{i}$ be the infimum of $a$ and the $\varepsilon$ with a $(d, \varepsilon)$ amalgamation of length less than or equal to $i$, and let $\zeta^{i}=\left\{\zeta_{j}^{i}\right\}$ be this amalgamation. Fixing $\varepsilon$ and varying $\xi$ as, say, $\xi(t)$, one sees that if $\zeta^{i}=\zeta^{i}(0)$ is the maximal amalgamation for $\xi=\xi(0)$ at $\varepsilon$, then it is an amalgamation for nearby $\xi(t)$, but it might not be maximal. It follows that the $\varepsilon_{i}$ vary continuously with $\xi$, but the $\zeta^{i}$ do not, in that $\zeta^{i}(0)$ might have smaller length than $\zeta^{i}(t)$ (in which case some of the $\varepsilon_{i}$ coincide).

The rough picture one can have is as follows. For each point $x_{n}$ present in the sum $\xi=\sum a_{n} x_{n} \in S P^{n}(X)$, one has a disk centred at $x_{n}$, whose size depends on $a_{n}$. The diameter increases with $\varepsilon$, and when two or more disks get too close, they get replaced by a bigger disk which includes them, with centre the centre of gravity of the points. This disk keeps on growing, until it meets another disk, etc. The final result is $r(\xi)$ disks, at $\varepsilon=a$.

Lemma (3.6). Fixing $d(k)$ as above, for all $\varepsilon, \delta>0$ sufficiently small, there exist continuous "concentration" maps

$$
f_{N}: S P^{N}(X) \rightarrow S P^{N}(X)
$$

with the following properties.

(a) If the maximal $(d, \varepsilon)$ amalgamation of $\xi \in S P^{N}(X)$ is

$$
\left\{\zeta_{j}\right\}, \quad \zeta_{j} \in S P^{n_{j}}(X), j=1, \ldots, r,
$$

then setting $\eta=\left(n_{1}, \ldots, n_{r}\right)$,

$$
f_{N}(\xi) \in \bigcup_{\eta^{\prime}>\eta} S_{\eta^{\prime}}
$$


where $S_{\eta}$ are the multiplicity strata of the symmetric product. (Thus also, $f_{N}(\xi) \in \bigcup_{\eta^{\prime} \geq \eta} S_{\eta}^{\prime}$ for $\left.\xi \in S_{\eta}.\right)$

(b) If the maximal $(d, \varepsilon+\delta)$ amalgamation of $\xi \in S P^{N}(X)$ is

$$
\left\{\phi_{k}\right\}, \quad \phi_{k} \in S P^{m_{k}}(X), k=1, \ldots, s,
$$

with centres of gravity $\bar{\phi}_{k}$, then $f_{N}$ maps the points lying in $\phi_{k}$ to the $(\varepsilon+\delta) d\left(m_{k}\right)$ ball centred at $\bar{\phi}_{k}$. If the maximal $(d, \rho)$ amalgation of $\xi$ is constant for all $\rho \in[\varepsilon, \varepsilon+\delta]$, then,

$$
f_{N}(\xi)=\sum_{k} m_{k} \bar{\phi}_{k}
$$

(c) Scaling $\varepsilon \delta$ to zero by $t \varepsilon, t \delta, t \in[0,1]$, gives a homotopy $f_{N, t}$ between $f_{N}$ and the identity map. This allows one to follow individual points $x_{i}$ of $\xi$ as they move to their image in $f_{N}(\xi)$. The paths taken by these points all lie within the $(\varepsilon+\delta) d\left(m_{k}\right)$ ball centred at the $\bar{\phi}_{k}$ corresponding to the point $x_{i}$, and all the points in $\zeta_{j}$ move to the same point.

(d) If $V_{N}(y), y \in X$ is the subset of $S P^{N}(X)$ of configurations with no point of $X$ within $3(\varepsilon+\delta)(d(N)+d(1))$ of $y$, then one has a commuting diagram

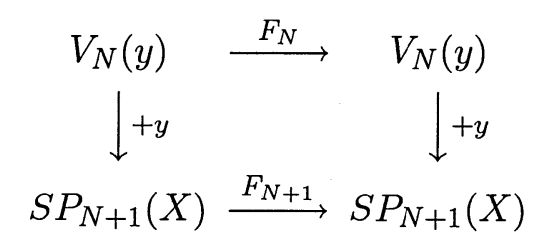

Proof. The idea of this map is that it moves the points in $\zeta_{j}$ to its centre of gravity $\bar{\zeta}_{j}$, and then perhaps concentrates them a bit more if there are further amalgamations in the interval $[\varepsilon, \varepsilon+\delta]$.

Let $\left(\varepsilon_{i}, \zeta^{i}=\left\{\zeta_{j}^{i}\right\}\right)$ be the amalgamation sequence of $\xi=\sum a_{n} x_{n}, x_{n} \in$ $X, a_{n} \in \mathbb{Z}^{+}$, for the window $[0, \varepsilon+\delta]$. Let us restrict this to consider only the $\varepsilon_{i}$ 's lying in the interval $(\varepsilon, \varepsilon+\delta)$, and renumber them as $\varepsilon_{1} \leq \cdots \leq \varepsilon_{k-1}$, setting $\varepsilon_{0}=\varepsilon$, and $\varepsilon_{k}=\varepsilon+\delta$. Let $\bar{\zeta}_{j}^{i}$ be the centers of gravity of the $\zeta_{j}^{i}$. Set $\zeta_{j\left(x_{n}\right)}^{i}$ to be the (nested) sequence of elements of $S P^{k_{j}^{i}}(X)$ to which $x_{n}$ belongs. Let us suppose for a moment that $X=\mathbb{R}^{k}$. Set

$$
w_{n}=w_{n}\left(x_{n}\right)=\sum_{i=1}^{k} \frac{\left(\varepsilon_{i}-\varepsilon_{i-1}\right)}{\delta} \bar{\zeta}_{j\left(x_{n}\right)}^{i-1}
$$


where the sum is the $\mathbb{R}^{k}$-sum, not the symmetric product sum. One then writes (using the symmetric product sum)

$$
f_{N}(\xi)=\sum a_{n} w_{n}
$$

From our discussion of the amalgamation sequences, this is continuous, and one checks that it has the right properties (e.g., if $\varepsilon_{1}=\varepsilon+\delta$, so that the amalgamation sequence is constant on $[\varepsilon, \varepsilon+\delta]$, then $\left.f_{N}(\xi)=\sum_{n} a_{n} \bar{\zeta}_{j\left(x_{n}\right)}^{0}\right)$.

The formula above for $w_{n}$ is simply that of the centre of gravity of the $\bar{\zeta}_{j\left(x_{n}\right)}^{i-1}$, weighted by the factors $\frac{\left(\varepsilon_{i}-\varepsilon_{i-1}\right)}{\delta}$. For a general $X$, we then use this same centre of gravity.

\section{3.b. Local flattenings.}

We now assume that we are on a compact subset $C$ (here $X \backslash Y$ ) of a Riemannian manifold $(X, g)$; let $3 R$ be the injectivity radius. On any geodesic ball $B_{r}\left(X, x_{0}\right)$ of radius $r$ less than $R$, centre $x_{0}$ in $C$, there is a natural way to "flatten" $g$. We consider the exponential map

$$
\varphi: B_{2 r}\left(T_{x_{0}}(X), 0\right) \rightarrow B_{2 r}\left(X, x_{0}\right) .
$$

This is a diffeomorphism, and we use it to define a flat metric $\left(\varphi^{-1}\right)^{*} g\left(x_{0}\right)$ on $B_{2 r}\left(X, x_{0}\right)$. Let us call this the geodesic metric centred at $x_{0}$. One can then patch this in to the metric $g$ using a partition of unity to get a global metric which is flat on $B_{r}\left(X, x_{0}\right)$.

Now let us consider $\xi=\sum a_{i} x_{i} \in S P^{N}(X)$. We would like to flatten the metric on balls containing the $x_{i}$, continuously in $\xi$. More precisely, let $\zeta_{j} \in S P^{m_{j}}(X)$ be the elements of the maximal $(d, 2 \delta)$ amalgamation of $\xi$, with centre of gravity $\overline{\zeta_{j}}$. We can flatten the metric on balls centred at the $\overline{\zeta_{j}}$, and of radius at least $2 \delta d\left(m_{j}\right)$, if we choose $\delta$ sufficiently small so that all our balls lie within the injectivity radius and so that the centres of gravity and the concentration maps are well defined.

Let $\left\{\tau_{j}\right\}, \tau_{j} \in S P^{n_{j}}(X)$ be the maximal $(d, 3 \delta)$-amalgation of $\xi$, with centres of gravity $\bar{\tau}_{j}$. The concentration map $f_{N}=f_{N}(3 \delta, 4 \delta)$ associates each point $x_{i}$ of $\xi$ to a $\bar{\tau}_{j}$, and then possibly moves $\bar{\tau}_{j}$ to a $\tilde{\tau}_{j}$. For each $x_{i}, x_{k}, i \neq k$, either the corresponding $\tilde{\tau}_{i}, \tilde{\tau}_{k}$ are the same, or the balls

$$
D_{9 \delta d\left(m_{i}\right)}\left(X, \bar{\tau}_{i}\right), \quad D_{9 \delta d\left(m_{k}\right)}\left(X, \bar{\tau}_{k}\right)
$$

are disjoint. In the latter case, the balls

$$
D_{4 \delta d\left(m_{i}\right)}\left(X, \bar{\zeta}_{i}\right), \quad D_{4 \delta d\left(m_{k}\right)}\left(X, \bar{\zeta}_{k}\right)
$$


are also disjoint.

Lemma (3.8). For all $\delta>0$, sufficiently small, there exists a continuous function

$$
\rho:(X)^{N} \longrightarrow\left(\mathbb{R}_{+}\right)^{N}
$$

which is equivariant with respect to the action of the symmetric group, and such that if $\xi=\sum a_{i} x_{i}$ has $\zeta_{j} \in S P^{m_{j}}(X)$ as its maximal $(d, 2 \delta)$ amalgamation, and $Z_{k} \in S P^{M_{\ell}}(X)$ as its maximal $(d, 3 \delta)$ amalgamation then:

(1) $\rho(x)_{i}$ is the same for all $x_{i}$ in $\zeta_{j}$.

(2) For $x_{i}$ in $\zeta_{j}, Z_{\ell}$,

$$
2 \delta d\left(m_{j}\right) \leq \rho(x)_{i} \leq 2 \delta d\left(M_{\ell}\right)
$$

Proof. As in the proof of $(3.6)$, let $\left(\delta_{i}, \zeta_{j}^{i}\right), i=0, \ldots, m$ be the amalgamation sequence for $\xi$ on the interval $[2 \delta, 3 \delta]$, with $\delta_{0}=2 \delta, \delta_{m}=3 \delta$. We set, for $x_{k} \in \zeta_{j\left(x_{k}\right)}^{i}, i=1, \ldots, m$

$$
\rho(x)_{k}=\sum_{i=1}^{m} 2\left(\delta_{i}-\delta_{i-1}\right) d\left(n_{j(x)}^{i}\right) .
$$

We then use this lemma to define bump functions which are one on the balls of radius $\rho(x)_{i}$, centred at the $\zeta$, zero outside the balls of radius $\rho(x)_{i}+\delta$. One then has that for each pair of points $x_{i}, x_{k}$ of $\xi$, that either the corresponding functions $\varphi_{i}, \varphi_{k}$ have disjoint support or that $x_{i}$ and $x_{k}$ correspond to the same point in $f_{N}(x)$. Let $\mu$ be a positive function which is one on the complement of the $\rho\left(x_{i}\right)+\delta$ balls and zero on the $\rho\left(x_{i}\right)$ balls, such that $\left(\sum_{i} \varphi_{i}\right)+\mu$ is bounded away from zero. These choices can be done continuously in $\xi$.

As remarked above, all the points in $\zeta_{j}$ correspond to the same $\tilde{\zeta}_{j}$ in $f_{N}(x)$. Let $g_{j}$ the geodesic metric centred at $\tilde{\zeta}_{j}$, and set

$$
\tilde{g}=\frac{\left(\sum_{j}\left(\varphi_{j} g_{j}\right)\right)+\mu g}{\left(\sum_{j} \varphi_{j}\right)+\mu}
$$

where the sum is taken from 1 to $n$ over the points $x_{i}$ of $\xi$, repeating if $x_{i}$ is multiple. This is the desired metric, and varies continuously with $\xi$. We 
note that the centres for defining the geodesic metric are not necesseraily in the balls of radius $\rho\left(x_{i}\right)$, and indeed are defined in such a way that if two of these balls come together, the center used to define the metric is already the same on both balls, so that the resulting metric stays flat.

\section{Instantons and connections on $\mathbb{R}^{4}$.}

\section{4.a. Instantons on $\mathbb{R}^{4}$.}

Let $X$ be a compact Riemannian manifold. The Yang-Mills functional for connections on $E_{k} \rightarrow X$ :

$$
\mathcal{Y} \mathcal{M}(A)=\int_{X}\left|F_{A}\right|^{2}
$$

has as minima the anti-self-dual connections (instantons), whose curvature $F_{A}$ satisfies the equation

$$
* F_{A}=-F_{A}
$$

One then has the framed moduli space $\mathcal{M}_{k}(X, p)$ of gauge equivalence classes of pairs $(A, T)$, where $A$ is an anti self-dual connection and $T$ is a trivialisation of $E_{k}$ over a base point $p$. For $X=S^{4}, \mathcal{M}_{k}\left(S^{4}, p\right)$ is a smooth $8 k$-dimensional manifold. It lies in the space $\mathcal{B}\left(S^{4}, p\right)$ of all pairs $(A, T)$ of pairs

$$
\text { (connections, trivialisations over } p \text { ), }
$$

which, in turn, is homotopy equivalent to $\mathcal{B}_{k}\left(S^{4}, D\right)$, where $D$ is a ball containing $p$. One has the theorem:

Theorem (4.3) (Atiyah-Jones conjecture) [BHMM, Ti1, Ti2, Ki]. For gauge groups $G=S U(n), S O(n), S p(n)$, the inclusion

$$
\mathcal{M}_{k}\left(S^{4}, p\right) \rightarrow \mathcal{B}_{k}\left(S^{4}, p\right)
$$

induces isomorphisms

$$
\pi_{i}\left(\mathcal{M}_{k}\left(S^{4}, p\right)\right) \simeq \pi_{i}\left(\mathcal{B}_{k}\left(S^{4}, p\right)\right) .
$$

for $i<[k / 2]-1$. 
The spaces $\mathcal{M}_{k}=\mathcal{M}_{k}\left(S^{4}, p\right)$ will then, in the limit, provide a homotopy model for $\mathcal{B}_{k}\left(S^{4}, p\right)$, and, as we shall see, consist (up to homotopy) of concentrated, particle-like connections which are not quite flat, however, far from the "particles". We will modify the connections so that they are flat outside disks, to obtain a space $\mathcal{P}_{k}\left(S^{4}\right)$ which is homotopic to $\mathcal{M}_{k}\left(S^{4}, p\right)$. The connections in $\mathcal{P}_{k}\left(S^{4}\right)$ can then be easily grafted or glued into any four-manifold.

We note that the Yang Mills functional and equation (4.2) are conformally invariant, so that solutions to (4.2) on $S^{4}$ are solutions on $\mathbb{R}^{4}$; indeed, a theorem of Uhlenbeck [U] tells us that $L^{2}$ solutions to $(4.2)$ on $\mathbb{R}^{4}$ are equivalent to solutions on $S^{4}$. The instantons of charge $k$ on $S^{4}$ are all given by the ADHM construction [ADHM] (see also [DK, ch. 3]). For gauge given $S U(n)$ this construction takes as input linear maps

$$
\tau_{1}, \tau_{2}: \mathbb{C}^{k} \rightarrow \mathbb{C}^{k}, \quad \sigma, \pi^{*}: \mathbb{C}^{n} \rightarrow \mathbb{C}^{k}
$$

and produces an instanton. As this construction is being presented to demonstrate the analytic behaviour of the instantons, it will suffice to think of other groups as being represented into $S U(n)$. Here $\mathbb{C}^{k}$ and $\mathbb{C}^{n}$ are equipped with their standard Hermitian metrics.

The matrices must satisfy the equations:

$$
\begin{gathered}
{\left[\tau_{1}, \tau_{2}\right]+\sigma \pi=0} \\
{\left[\tau_{1}, \tau_{1}^{*}\right]+\left[\tau_{2}, \tau_{2}^{*}\right]+\sigma \sigma^{*}-\pi^{*} \pi=0}
\end{gathered}
$$

as well as some non-degeneracy conditions: set $z_{1}=x_{1}+i x_{0}, z_{2}=x_{3}+i x_{4}$, and define :

$$
\alpha_{x}^{*}=\left(\tau_{1}^{*}-\bar{z}_{1}, \tau_{2}^{*}-\bar{z}_{2}, \pi^{*}\right), \quad \beta_{x}=\left(-\tau_{2}+z_{2}, \tau_{1}-z_{1}, \sigma\right)
$$

then the map

$$
R_{x}=\alpha_{x}^{*}+\beta_{x}: \mathbb{C}^{k} \oplus \mathbb{C}^{k} \oplus \mathbb{C}^{n} \rightarrow \mathbb{C}^{k} \oplus \mathbb{C}^{k}
$$

must be surjective for all $x$. The kernel $K_{x}$ of $R_{x}$ is then a subbundle of the trivial bundle $\mathbb{C}^{k} \oplus \mathbb{C}^{k} \oplus \mathbb{C}^{n}$, and one obtains a connection on $K_{x}$ by orthogonal projection of the standard flat connection on $\mathbb{C}^{k} \oplus \mathbb{C}^{k} \oplus \mathbb{C}^{n}$. Explicitly, if

$$
M=M(x): \mathbb{C}^{n} \rightarrow \mathbb{C}^{k} \oplus \mathbb{C}^{k} \oplus \mathbb{C}^{n}
$$


provides a basis (not necessarily Hermitian) for the kernel $K_{x}$, then $M\left(M^{*} M\right)^{-\frac{1}{2}}$ gives a Hermitian basis and the connection matrix is given by

$$
A=d\left(M^{*} M\right)^{\frac{1}{2}}\left(M^{*} M\right)^{-\frac{1}{2}}+\left(M^{*} M\right)^{-\frac{1}{2}} M^{*} d M\left(M^{*} M\right)^{-\frac{1}{2}}
$$

and the curvature by

$$
F_{A}=\left(M^{*} M\right)^{-\frac{1}{2}}\left[d M^{*}\left(1-M\left(M^{*} M\right)^{-1} M^{*}\right) d M\right]\left(M^{*} M\right)^{-\frac{1}{2}}
$$

The equations (4.6) guarantee that these are instantons. The group $U(k)$ acts on the matrices by

$$
\left(\tau_{1}, \tau_{2}, \sigma, \pi\right) \mapsto\left(g \tau_{1} g^{-1}, g \tau_{2} g^{-1}, \sigma g^{-1}, g \pi\right)
$$

and one has the theorem:

Theorem (4.12)[ADHM]. $\mathcal{M}_{k}$ is the quotient of the variety of matrices $\left(\tau_{1}, \tau_{2}, \sigma, \pi\right)$ satisfying equations (4.6) and the non-degeneracy condition (4.8) by the group $U(k)$.

The construction is quite explicit, and one can show that the curvature tends to concentrate around the points in $\mathbb{R}^{4}$ corresponding to the eigenvalues of the matrices $\left(\tau_{1}, \tau_{2}\right)$, in particular when $\sigma$ and $\pi$ are small, so that $\tau_{1}, \tau_{2}$ almost commute.

Let us write $R_{x}$ as

$$
R_{x}=\left(N_{x}, P\right)
$$

where $P$ is $2 k \times n, N_{x}=\left(N_{0}-\mathbb{I}_{k \times k} \otimes \gamma \cdot x\right)$ is $2 k \times 2 k$, with $\gamma \cdot x$ the representation of the quaternion $x$ as a $2 \times 2$ matrix. Far from the origin, $N_{x}$ is invertible, and we can set

$$
M=\left(\begin{array}{c}
Q \\
\mathbb{I}_{n \times n}
\end{array}\right), \quad Q=-\left(N_{x}^{-1}\right) P
$$

so that $M$ provides a basis for the kernel $K_{x}$. One then has for the connection

$$
A=\left(d\left[\mathbb{I}+Q^{*} Q\right]^{\frac{1}{2}}\right)\left[\mathbb{I}+Q^{*} Q\right]^{-\frac{1}{2}}+\left[\mathbb{I}+Q^{*} Q\right]^{-\frac{1}{2}} Q^{*} d Q\left[\mathbb{I}+Q^{*} Q\right]^{-\frac{1}{2}}
$$

and for the curvature:

$$
F_{A}=\left[\mathbb{I}+Q^{*} Q\right]^{-\frac{1}{2}}\left(d Q^{*}\right)\left(1-Q\left[\mathbb{I}+Q^{*} Q\right]^{-1} Q^{*}\right)(d Q)\left[\mathbb{I}+Q^{*} Q\right]^{-\frac{1}{2}}
$$

Note that as $x$ gets large, $Q$ is $O\left(x^{-1}\right)$ and $d Q$ is $O\left(\left|x^{-2}\right|\right)$. 
Recalling that the equations are conformally invariant, one has that the action of the dilation $\mathcal{D}_{\lambda}$ with centre 0 , factor $\lambda$ on $\mathbb{R}^{4}$, acts on the moduli space by:

$$
\left(\tau_{1}, \tau_{2}, \sigma, \pi\right) \mapsto \lambda\left(\tau_{1}, \tau_{2}, \sigma, \pi\right)
$$

and similarily on $N_{0}, P$. For $\lambda<1$ this concentrates the connection around the origin.

We define a space $\mathcal{M}_{k}^{c}=\mathcal{M}_{k}^{c}(\varepsilon, \delta)$ of concentrated instantons by the properties:

1. The operator norm of $N_{0}$ is less than $3 \varepsilon d(k) / 2$

2. The operator norm of $P$ is less than $\delta$

Proposition (4.16). (a) $\mathcal{M}_{k}^{c}$ is a strong deformation retract of $\mathcal{M}_{k}$.

(b) $\mathcal{M}_{k}^{c}$ is invariant under dilations by a factor $\lambda<1$.

(c) There is a constant $C$ such that outside the ball of radius $2 \varepsilon d(k)$ the $L^{\infty}$ norms of $A$ is bounded by $C \delta|x|^{-3}$, that of $F_{A}$ by $C \delta|x|^{-4}$, and the $L^{2}$ norm of $F_{A}$ by $C \delta$.

(d) One can define a function $\rho: \mathcal{M}_{k}^{c} \rightarrow(0,2 \epsilon d(k))$, equivariant with respect to the action of dilations, such that the instanton $A$ has all but $C \delta$ of the $L^{2}$-norm of the curvature concentrated in a ball of radius $\rho(A)$ around the origin.

Proof. For (c), one has that outside the sphere of radius $2 \varepsilon d(k)$, the norm of $Q$ is bounded by $C \cdot|x|^{-1}$, and that of $d Q$ by $C \cdot|x|^{-2}$ for some suitable constant $C$. The estimates then follow from the formulae (4.14), (4.15). Parts (a), (b), (d) then simply follow from the action of the dilation on the ADHM matrices.

Underlying much of gauge theory is a technique due to Taubes for glueing two or more instantons which are concentrated at different well separated points: one first patches them together using a partition of unity, and then deforms the result to an instanton. We will now use this construction with our concentrated instantons as building blocks to build a space of concentrated particle-like connections. In our version, we will first consider an element $\xi=\sum_{i} m_{i} x_{i}$ of $S P^{k}\left(\mathbb{R}^{4}\right)$ whose maximal $(d, \varepsilon)$ amalgamation is minimal, i.e. $\zeta_{i}=m_{i} x_{i}$. This guarantees that $x_{i}$ and $x_{j}$ are separated by $3 \varepsilon\left(d\left(m_{i}\right)+d\left(m_{j}\right)\right)$. On small balls containing the $x_{i}$, we will glue in to a trivial background connection $\Gamma$ elements $A_{i}$ of $\mathcal{M}_{m_{i}}^{c}=\mathcal{M}_{m_{i}}^{c}\left(\mathbb{R}^{4}\right)$, as 
follows. Choosing an orthonormal frame at the $x_{i}$ we obtain via the exponential map an identification between $D_{3 \varepsilon d\left(m_{i}\right)}\left(\mathbb{R}^{4}, x_{i}\right)$ and $D_{3 \varepsilon d\left(m_{i}\right)}\left(\mathbb{R}^{4}, 0\right)$ (there is a canonical choice of frame for $\mathbb{R}^{4}$, but we will be doing this for other manifolds). We choose a radial gauge for $A_{i}$, with centre at infinity in $S^{4}$, the pole opposite to the one at which the instanton is concentrated. This gauge allows one to identify the bundle $E_{k}$ over $D_{3 \varepsilon d\left(m_{i}\right)}\left(\mathbb{R}^{4}, 0\right) \backslash\{0\}$ with the trivial bundle over $D_{3 \varepsilon d\left(m_{i}\right)}\left(\mathbb{R}^{4}, x_{i}\right) \backslash\left\{x_{i}\right\}$, in such a way that $x_{i}$ corresponds to 0 . One fixes a bump function function $\phi$ which is one on $D_{1}\left(\mathbb{R}^{4}, 0\right)$ and vanishes outside $D_{3 / 2}\left(\mathbb{R}^{4}, 0\right)$, with gradient bounded by 4 , rescales it to the radius $\rho\left(A_{i}\right)<2 \varepsilon d\left(m_{i}\right)$ of $(4.16)$ and centres it at the $x_{i}$ : let $\phi_{i}$ denote the resulting function. One then sets, over $D_{3 \varepsilon d\left(m_{i}\right)}\left(\mathbb{R}^{4}, x_{i}\right) \backslash\left\{x_{i}\right\}$,

$$
\hat{A}=\left(1-\phi_{i}\right) \Gamma+\phi_{i} A_{i}
$$

where $\Gamma$ is the product connection over the trivial bundle and $A_{i}$ is the representative for $\phi_{i}$ in the radial gauge. The result is a connection which is trivial on the complement of balls, and indeed trivialised on that set.

This connection is almost anti-self-dual, and for a suitable choice of $\delta$ can be deformed to an anti-self-dual connection. Indeed, from the properties (4.16) of our concentrated connections, the $L^{2}$ norm of the self dual part of the curvature of $\hat{A}$ is small, and with appropriate choices of constants in (4.16) can be made as uniformly small as one wishes. One can then appeal to the following theorem of Taubes. Let $I$ be the group of conformal automorphisms of $\mathbb{R}^{4}$ generated by the rotations and the dilations centred at the origin.

Theorem (4.17). Let $\mathcal{B}_{k}^{\delta}$ be the space of based equivalence classes of connections on $E_{k} \rightarrow S^{4}$ whose anti-self dual curvature has $L^{2}$ norm less than $\delta$. For $\delta$ sufficiently small, there is a strong deformation retraction $R_{k}^{t}$ of $\mathcal{B}_{k}^{\delta}$ onto $\mathcal{M}_{k} .\left(R_{k}^{0}=I d, R_{k}^{1}\left(\mathcal{B}_{k}^{\delta}\right)=\mathcal{M}_{k}\right)$. This retraction is I-equivariant.

This result, apart from the invariance, is proven in [T1], over the four-sphere. To obtain the $I$-invariance, one must transfer the proof to $\mathbb{R}^{4}$, and indeed, this can be carried out [T4]; the key point is the conformal invariance of an appropriate Laplacian. Furthermore it follows by standard estimates (as in [DK, ch.7-8]) that the result is again concentrated, though indeed, if it were not, one could just build a concentration into the retraction. In any case, applying $R$ to $\hat{A}$, we obtain $A \in \mathcal{M}_{k}$.

The next step is to implement this glueing construction of particle-like connections supported in disks in some continuous fashion over $S P^{k}\left(\mathbb{R}^{4}\right)$. The Taubes retraction will be used when the disks get too close together to 
turn the almost instantons supported on small disks into an almost instanton on a bigger disk which contains them. We divide $S P^{k}\left(\mathbb{R}^{4}\right)$ into its multiplicity strata $\mathcal{S}_{\mu}, \mu=\left(m_{1}, m_{2}, \ldots, m_{s}\right), m_{1} \geq m_{2} \geq \cdots \geq m_{s}>0, \sum m_{i}=k$ :

$$
\mathcal{S}_{\mu}=\left\{\sum_{i=1}^{s} m_{i} x_{i} \in S P^{k}\left(\mathbb{R}^{4}\right), x_{i} \neq x_{j} \text { for } i \neq j\right\} .
$$

One defines for each of these strata a subspace $\mathcal{S}_{\mu}^{\varepsilon}$ consisting of those elements with well separated points. If $\mu=\left(m_{1}, m_{2}, \ldots, m_{s}\right)$, set $|\mu|=s$. $\mathcal{S}_{\mu}^{\varepsilon}$ will consist of those elements whose amalgamation sequence satisfies $\varepsilon_{i} \geq(k-i) \varepsilon$.

We now consider the space

$$
\begin{array}{r}
\mathcal{V}_{\mu}^{\varepsilon}=\left\{\left(x_{1}, \ldots, x_{s}\right),\left(f_{1}, \ldots, f_{s}\right),\left(A_{1}, \ldots, A_{s}\right) \mid \sum m_{i} x_{i} \in \mathcal{S}_{\mu}^{\varepsilon},\right. \\
\left.f_{i} \in F r_{x_{i}}, A_{i} \in \mathcal{M}_{m_{i}}^{c}\right\},
\end{array}
$$

where $F r_{x} \simeq S O(4)$ is the space of frames at $x$. We quotient this space by the simultaneous action of $S O(4)^{s}$ on the framings and the moduli spaces, to remove the redundancy given by the framings so that we only consider essentially different instantons, and further quotient by the subgroup $S y m_{\mu}$ of the symmetric group which stabilises $\mu$, so as to have unordered points, labelled by concentrated instantons, which are to be thought of as being glued in near the points. Let the quotient be

$$
\mathcal{P}_{k, \mu}^{\varepsilon}=\left[\mathcal{V}_{\mu}^{\varepsilon} /(S O(4))^{s}\right] / \text { Sym }_{\mu} .
$$

We perform the Taubes glueing construction on these spaces, so that the $\mathcal{P}_{k, \mu}^{\varepsilon}$ are embedded in $\mathcal{B}\left(S^{4}, p\right)$ as a space of concentrated connections, flat outside small balls.

These spaces will be the constituant strata of a space $\mathcal{P}_{k}^{\varepsilon}=\mathcal{P}_{k}^{\varepsilon}\left(S^{4}\right)$ which will turn out to be homotopy equivalent to $\mathcal{M}_{k}\left(S^{4}, p\right)$. We again have a partial ordering on the $\mathcal{P}_{k, \mu}^{\varepsilon}$ which corresponds to the ordering on the strata of the symmetric product, so that $\mu=(k)$ corresponds to the highest stratum and $\mu=(1,1,1, \ldots, 1)$, the lowest.

We will build $\mathcal{P}_{k}^{\varepsilon}$ in the spirit of a CW-complex, starting with the "smallest" stratum $(\mu=1)$ and glueing on the "bigger" ones. To illustrate what is involved, we will start with the case $k=3$. We have then three strata, labelled by $(3),(2,1),(1,1,1)$.

(a) Glueing the boundary of $\mathcal{P}_{3,(2,1)}^{\varepsilon}$ to $\mathcal{P}_{3,(3)}^{\varepsilon}$. (Note: "boundary" in fact refers here only to the part of the boundary corresponding to particles 
becoming close to each other, not, for example, to boundaries in the moduli spaces). Consider an element $A$ of $\mathcal{P}_{3,(2,1)}^{\varepsilon}$ lying over an element $\xi=2 x_{1}+x_{2}$ of $\mathcal{S}^{\varepsilon}{ }_{(2,1)}$, and consider the amalgamation sequence of $\xi$ : this is simply given by one number $\varepsilon_{1} \geq 2 \varepsilon$, at which the $(2,1)$ configuration amalgamates to a (3) configuration. If $\varepsilon_{1}<3 \varepsilon$, we use the Taubes retraction to retract the connection $A$ towards $\mathcal{M}_{3}$; assuming the full retraction takes place over $[0,1]$, we perform the retraction for a time of length $\left[3 \varepsilon-\varepsilon_{1}\right] / \varepsilon$. This procedure gives a connection which is no longer flat outside small balls, so we must re-flatten. The Taubes retraction moves us towards an instanton of charge three, and we use (4.16) to choose its associated radius $\rho$. One flattens the associated connection with a bump function built from our standard $\phi$ with radius $\rho$ and centre the centre of mass of the two points. Flattening the connections with this bump function lands the boundary $\varepsilon_{1}=2 \varepsilon$ of the stratum $\mathcal{P}_{3,(2,1)}^{\varepsilon}$ in $\mathcal{P}_{3,(3)}^{\varepsilon}$; let the resulting glueing of the two strata be denoted $\mathcal{P}_{3,2^{+}}^{\varepsilon}$.

(b) Glueing the boundary of $\mathcal{P}_{3,(1,1,1)}^{\varepsilon}$ to $\mathcal{P}_{3,2^{+}}^{\varepsilon}$. Let us next consider an element $A$ of $\mathcal{P}_{3,(1,1,1)}^{\varepsilon}$, lying over $x_{1}+x_{2}+x_{3}$ in $\mathcal{S}_{(1,1,1)}$. Its amalgamation sequence consists of two numbers $\varepsilon_{2} \leq \varepsilon_{1}$, with $\varepsilon \leq \varepsilon_{2}, 2 \varepsilon \leq \varepsilon_{1}$; if $\varepsilon_{2}<\varepsilon_{1}$, then one first amalgamates to a $(2,1)$ configuration, before going to the (3) configuration. If $\varepsilon_{2}<2 \varepsilon$, we first deform the instantons corresponding to the two points being amalgamated (say $x_{1}$ and $x_{2}$ ), towards an instanton of charge 2, using the Taubes retraction. We do this for a time $\left[2 \varepsilon-\varepsilon_{2}\right] / \varepsilon$. Again we reflatten outside balls, using the bump function with radius given by the function $\rho$ evaluated on the Taubes retraction of the two charge one connections, and with centre the centre of mass of $x_{1}$ and $x_{2}$. Next, if $\varepsilon_{1}<3 \varepsilon$, retract the result to the instantons of charge three, for a time $\left[3 \varepsilon-\varepsilon_{1}\right] / \varepsilon$, and again reflatten. At the boundary of the stratum, we then have one of two situations: either $\varepsilon_{2}=\varepsilon$, or $\varepsilon_{1}=2 \varepsilon$. In the first case, we have first retracted to $\mathcal{P}_{3,(2,1)}^{\varepsilon}$, and then possibly moved to $\mathcal{P}_{3,(3)}^{\varepsilon}$, using the recipe of (a) above. In the second case, we have deformed our element $A$ to an element of $\mathcal{P}_{3,(3)}^{\varepsilon}$. In any case, the boundary is now glued to $\mathcal{P}_{3,2^{+}}^{\varepsilon}$.

The general case proceeds in a similar fashion. One glues the edge of each stratum $\mathcal{P}_{k, \mu}^{\varepsilon}$ onto a space made out of the higher order strata. If $\xi \in \mathcal{P}_{k, \mu}^{\varepsilon}$, one considers its amalgamation sequence $\left(\varepsilon_{i}, \zeta_{i}\right)$, with $\varepsilon_{s} \leq \varepsilon_{s-1} \leq \cdots \leq \varepsilon_{1}$. If $\varepsilon_{s}<(k-s+1) \varepsilon$, we retract the subconfigurations being amalgamated towards the appropriate moduli space, for a time $\left[(k-s+1) \varepsilon-\varepsilon_{s}\right] / \varepsilon$, then reflatten. We then move on to $\varepsilon_{s-1}$ : if $\varepsilon_{s-1}<(k-s) \varepsilon$, retract for the appropriate time, and reflatten. One repeats this procedure all the way up 
to $\varepsilon_{1}$.

Proposition (4.21). The spaces $\mathcal{P}_{k}^{\varepsilon}$ and $\mathcal{M}_{k}$ are homotopy equivalent.

Proof. On each stratum $\mathcal{P}_{k, \mu}^{\varepsilon}$ there is a natural infinitesimal action of the contraction $x \mapsto c x, c<1$ towards the origin in $\mathbb{R}^{4}$, acting both on $S P^{k}\left(\mathbb{R}^{4}\right)$ and on the moduli spaces of instantons. This vector field is not complete, as particles eventually get too close to each other, with $\varepsilon_{i}<(k-i) \varepsilon$, and "fall off the edge". One now notes that all our glueings, retractions, radii for bump functions, etc. have been chosen to be equivariant with respect to this contraction, so that one can redefine the contraction so that instead of falling off the edge when one reaches the edge of a stratum, one falls into a higher stratum. Continuing in this way, one eventually falls into the highest stratum $\mathcal{P}_{k,(k)}^{\varepsilon}$, which is invariant under the contraction. In other words, one has a deformation retraction of $\mathcal{P}_{k}^{\varepsilon}$ to $\mathcal{P}_{k,(k)}^{\varepsilon}$, and the latter is homotopy equivalent to $\mathcal{M}_{k}$, by the Taubes retraction.

Instead of considering particles over $\mathbb{R}^{4}$, we can only consider those configurations in $S_{\mu}$ which lie inside a suitably large ball $D^{4}$. This gives us a space

$$
\mathcal{P}_{k}^{\varepsilon}\left(S^{4}, D^{4}\right)=\mathcal{P}_{k}^{\varepsilon}\left(D^{4}, \hat{\partial} D^{4}\right)
$$

which will be our starting point for building other particle spaces. We will see that $\mathcal{P}_{k}^{\varepsilon}\left(D^{4}, \hat{\partial} D^{4}\right)$ is homotopy equivalent to $\mathcal{P}_{k}^{\varepsilon}$, for $\varepsilon$ sufficiently small.

\section{Particles and connections on a pair.}

\section{5.a. Particle spaces.}

Let us now return to our pairs $(X, Y)$, and define a suitable space of connections for these, based on what we have already done on $\mathbb{R}^{4}$. We assume that we have chosen a suitable metric on $X$.

As above, we consider the partition of $S P^{k}(X \backslash Y)$ into strata:

$$
\mathcal{S}_{\mu}(X, Y)=\left\{\sum_{i=1}^{s} m_{i} x_{i} \in S P^{k}\left(X \backslash Y_{0}\right), x_{i} \neq x_{j} \text { for } i \neq j\right\} .
$$

We first perform the flattening construction of section 3.b on our metric, which in a neighbourhood of each element of $\mathcal{S}_{\mu}(X, Y)$ gives us in a continuous way a flat metric with which to work. We will assume that the ratio $\delta / \varepsilon$ 
of the parameter $\delta$ associated with the flattenings to the parameter $\varepsilon$ associated to the scale of the bump-like connections is sufficiently large for all constructions associated with the glueing of our strata to place well within the flattened locus. We must choose both $\delta$ and $\varepsilon$ sufficiently small so that

- The flattening construction performed above remains within the injectivity radius of the manifold.

- The constructions involving centres of gravity are well defined.

- The width of the "collar" $Y_{1 / 2} \backslash Y_{0}$ is greater than the radii involved in all our constructions, so that all our "particles" will lie in the complement of $Y_{0}$.

- The ratio of the flattened metrics to the actual one is close to one.

Now define $\hat{\mathcal{S}}_{\mu}^{\varepsilon}(X, Y)$ to consist of those elements of $\mathcal{S}_{\mu}(X, Y)$ whose amalgamation sequence in the flattened metric satisfies $\varepsilon_{i} \geq(k-i) \varepsilon$. We define a location map $L_{\mu}: \hat{\mathcal{S}}_{\mu}^{\varepsilon}(X, Y) \rightarrow S P^{k}(X)$ which is similar to the concentration map of (3.4). More precisely, for $\xi=\sum_{j=1}^{s} m_{j} x_{j} \in \mathcal{S}_{\mu}^{\varepsilon}(X, Y)$, set $t_{i}=\varepsilon^{-1} \min \left(0,(k-i+1) \varepsilon-\varepsilon_{i}\right)$ for each $\varepsilon_{i}$ in the amalgamation sequence; note that $t_{i} \in[0,1)$, with $t_{i}=0$ for points far from the edge of $\mathcal{S}_{\mu}^{\varepsilon}(X, Y)$, and with the boundary of $\mathcal{S}_{\mu}^{\varepsilon}(X, Y)$ corresponding to one of the $t_{i}$ being 1. Now for each $x_{j}$, let $x_{j, s}=x_{j}, x_{j, s-1}, \ldots, x_{j, 1}$ be the centres of mass of the successive amalgamations to which $x_{j}$ belongs. Set, by descending induction,

$$
c_{j, s}=x_{j, s}, \quad c_{j, i}=C M\left(\left(c_{i+1}, 1-t_{i}\right),\left(x_{j, i}, t_{i}\right)\right)
$$

where $C M((y, s),(x, t))$ denotes the centre of mass of $y$ and $x$, weighted by the factors $s$ and $t$ respectively. We set $L_{\mu}\left(\sum m_{j} x_{j}\right)=\sum m_{j} c_{j, 1}$. If the collar is wide enough, then the inverse image under $L_{\mu}$ of any configuration containing a point of $Y_{0}$ must contain a point of $Y_{1 / 2}$. We will suppose that this is the case. 
Now put

$$
\mathcal{S}_{\mu}^{\varepsilon}(X, Y)=\left\{\sum m_{i} x_{i} \in \hat{\mathcal{S}}_{\mu}^{\varepsilon}(X, Y) \mid L_{\mu}\left(\sum m_{i} x_{i}\right) \in S P^{k}\left(X \backslash Y_{1 / 2}\right)\right\},
$$

$$
\begin{aligned}
\mathcal{V}_{\mu}^{\varepsilon}(X, Y)=\left\{\left(x_{1}, \ldots, x_{s}\right),\left(f_{1}, \ldots, f_{s}\right),\left(A_{1}, \ldots, A_{s}\right) \mid\right. \\
\left.\sum m_{i} x_{i} \in \mathcal{S}_{\mu}^{\varepsilon}(X, Y), f_{i} \in F r_{x_{i}}, A_{i} \in \mathcal{M}_{m_{i}}^{c}\right\}
\end{aligned}
$$

$$
\mathcal{P}_{k, \mu}^{\varepsilon}(X, Y)=\left[\mathcal{V}_{\mu}^{\varepsilon}(X, Y) /\left(S O(4)^{s}\right] / S y m_{\mu}\right.
$$

Over the flattened locus, we can perform all of our glueing of instanton operations as if they were in $\mathbb{R}^{4}$, using the isomorphisms given by the framings $f_{i}$ and the exponential maps of the flattened metrics. As long as we stay within an area on which the metric is flat, the identifications we make with $\mathbb{R}^{4}$ are unambiguous, up to a rotation and a translation, and our construction is equivariant with respect to these. We then obtain a space of particles $\mathcal{P}_{k}^{\varepsilon}(X, Y)$. The connections in our particle spaces are all flat, and indeed trivial and trivialised, over the complements of balls in $X \backslash Y$. In particular they are trivialised on $Y$, and so define elements $(\alpha, t)$ of $\mathcal{B}(X, Y)$.We note that there is a natural location map

$$
L: \mathcal{P}_{k}^{\varepsilon}(X, Y) \rightarrow S P^{k}(X)
$$

which, on the strata $\mathcal{P}_{k, \mu}^{\varepsilon}(X, Y)$ is the composition of the projection to $\mathcal{S}_{\mu}^{\varepsilon}(X, Y)$ with the concentration map $L_{\mu}: \mathcal{S}_{\mu}^{\varepsilon}(X, Y) \rightarrow S P^{k}(X)$. Indeed, at each step of the construction of $\mathcal{P}_{k}^{\varepsilon}(X, Y)$, we glued the edges of successive strata to the higher ones using the Taubes retraction towards instantons located at the successive centres of gravity of the amalgamation sequence of our element of $\mathcal{S}_{\mu}^{\varepsilon}(X, Y)$. Following the definition of the concentration map, we just have to define the location of the instanton as a suitably weighted average of these centres of gravity. This is done above for the strata $\mathcal{P}_{k, \mu}^{\varepsilon}(X, Y)$ via the map $L_{\mu}$. From the definitions of the glueings of the strata, $L$ is continuous, and behaves sensibly, so that for example, $L\left(\sum m_{i} x_{i}, f_{i}, A_{i}\right)=\sum m_{i} x_{i}$ away from the edges of the stratum.

The $\mathcal{P}_{k}^{\varepsilon}(X, Y)$ have several useful properties:

\section{a) Metric invariance.}


Proposition 5.5. Given any two metrics $g_{0}, g_{1}$, for $\varepsilon>0$ sufficiently small, the spaces $\mathcal{P}_{k}^{\varepsilon}(X, Y)_{0}=\mathcal{P}_{k}^{\varepsilon}(X, Y)\left(g_{0}\right)$ and $\mathcal{P}_{k}^{\varepsilon}(X, Y)_{1}=\mathcal{P}_{k}^{\varepsilon}(X, Y)\left(g_{1}\right)$ are homotopy equivalent.

Proof. As all the glueings are performed in $\mathbb{R}^{4}$, it will suffice to define equivalences $H_{\mu}$ between the strata $\mathcal{S}_{\mu}^{\varepsilon}(X, Y)\left(g_{0}\right)$ and $\mathcal{S}_{\mu}^{\varepsilon}(X, Y)\left(g_{1}\right)$ which, near the boundary (where the glueings are being done), are such that $x$ and $H_{\mu}(x)$ correspond to the same configuration in $\mathcal{S}_{\mu}^{\varepsilon}\left(\mathbb{R}^{4}\right)$. As the $\mathcal{V}_{\mu}^{\varepsilon}(X, Y)$ fiber over the $\mathcal{S}_{\mu}^{\varepsilon}(X, Y)$ one can then make the obvious identification, in a way that is compatible with all the glueings. Recall from (3.4) the concentration map $F_{k}: \mathcal{S}_{\mu}^{\varepsilon}(X, Y)\left(g_{0}\right) \rightarrow S P^{k}(X, Y)$, with parameters $(\delta, \delta)$, and consider as above for each $\left(\sum m_{i} x_{i}\right)$ the flattened metrics built from $g_{0}, g_{1}$ on balls which for each $x_{i}$ contain $x_{i}$ and the point $y_{j}$ in $F_{k}\left(\sum m_{i} x_{i}\right)$ corresponding to it. We are assuming as usual that the flattening parameter $\delta$ is considerably larger than $2 k \varepsilon$. We use the points $y_{j}$ in $F_{k}\left(\sum m_{i} x_{i}\right)$ as centres, and, choosing for each $y_{j}$ a frame in the tangent space at that point, we then define two local isometries $\phi_{0}, \phi_{1}$ of $X$ with $\mathbb{R}^{4}$, one using the flattened $g_{0}$ metric, the other the flattened $g_{1}$ metric.

$$
X \leftarrow T_{y_{0}} \rightarrow X
$$

The composition $\phi_{1}^{-1} \phi_{0}$ is independent of the frame chosen and associates to $\left(\sum m_{i} x_{i}\right),\left(f_{1}, \ldots, f_{s}\right)$ a corresponding element $H_{\mu}\left(\left(\sum m_{i} x_{i}\right),\left(f_{1}, \ldots, f_{s}\right)\right)$, in such a way that the amalgamation sequences of corresponding points are the same, at least up to $2 k \varepsilon$, and that distances between the points amalgamated by $F_{k}$ are the same for both $\left(\sum m_{i} x_{i}\right),\left(f_{1}, \ldots, f_{s}\right)$ and $H_{\mu}\left(\left(\sum m_{i} x_{i}\right),\left(f_{1}, \ldots, f_{s}\right)\right)$. (The reason $\varepsilon$ must be small is for the maps to be defined.)

Corollary (5.7). For $\varepsilon$ sufficiently small,

$$
\mathcal{P}^{\varepsilon}\left(D^{4}, \hat{\partial}\left(D^{4}\right)\right) \simeq \mathcal{P}_{k}^{\varepsilon} \simeq \mathcal{M}_{k}
$$

\section{b) Scale invariance.}

Corollary (5.8). The spaces $\mathcal{P}_{k}^{\varepsilon}(X, Y)$ have homotopy type which is independent of $\varepsilon$ for $\varepsilon$ sufficiently small.

One simply rescales the metric. We also remark that the $\mathcal{P}_{k}^{\varepsilon}(X, Y)$ are invariant under diffeomorphism, using the pull-back metric. 


\section{c) Loop sum.}

Let the support of the connection be the union of the closures of the balls over which the connections are not trivial. Now let us suppose that the supports of two connections $(\alpha, t) \in \mathcal{P}_{k}^{\varepsilon}(X, Y)$ and $(\beta, s) \in \mathcal{P}_{l}^{\varepsilon}(X, Y)$ are disjoint. Then the trivialisations allow us to identify the relevant bundles over the complement of the union of the support, and we obtain a connection $(\alpha, t) *(\beta, s)$

Proposition (5.9) (Loop sum). Let $V$ in $\mathcal{P}_{k}^{\varepsilon}(X, Y) \times \mathcal{P}_{l}^{\varepsilon}(X, Y)$ be the set of pairs with disjoint support. There is a well defined continuous "loop sum" map

$$
V \rightarrow \mathcal{P}_{k+l}^{\varepsilon}(X, Y)
$$

\section{d) Restriction and excision.}

We note that in general, the spaces $\mathcal{P}_{k}(X, Y)$ split into a family of connected components, corresponding to the different ways of placing particles in the different connected components of $X \backslash Y$, and so of partitioning $k$ into $\kappa=\left(k_{1}, k_{2}, \ldots, k_{s}\right)$, where $\sum k_{i}=k$ and $s$ is the number of connected components of $X \backslash Y$. Let $\mathcal{P}_{k, \kappa}(X, Y)$ denote these various components. Now let $Y^{\prime} \subset Y$; the inclusion $X \backslash Y \mapsto X \backslash Y^{\prime}$ induces maps $S P^{k}(X \backslash Y) \rightarrow S P^{k}\left(X \backslash Y^{\prime}\right)$. There are then induced inclusions

$$
I: \mathcal{P}_{k, \kappa}^{\varepsilon}(X, Y) \rightarrow \mathcal{P}_{k, \kappa^{\prime}}^{\varepsilon}\left(X, Y^{\prime}\right)
$$

(the multi-index might change as there might not be the same number of connected components.)

Furthermore, if $V$ is closed in $Y$ we see that the natural restriction map on trivialisations from $Y$ to $Y \backslash V$ defines an isomorphism

$$
E: \mathcal{P}_{k}^{\varepsilon}(X, Y) \rightarrow \mathcal{P}_{k}^{\varepsilon}(X \backslash V, Y \backslash V)
$$


Finally, if $\left(X_{1}, Y_{1}\right),\left(X_{2}, Y_{2}\right)$ are such that $X_{1} \backslash Y_{1}$ and $X_{2}$ are disjoint, and $X_{2} \backslash Y_{2}$ and $X_{1}$ are disjoint, we have that the composition

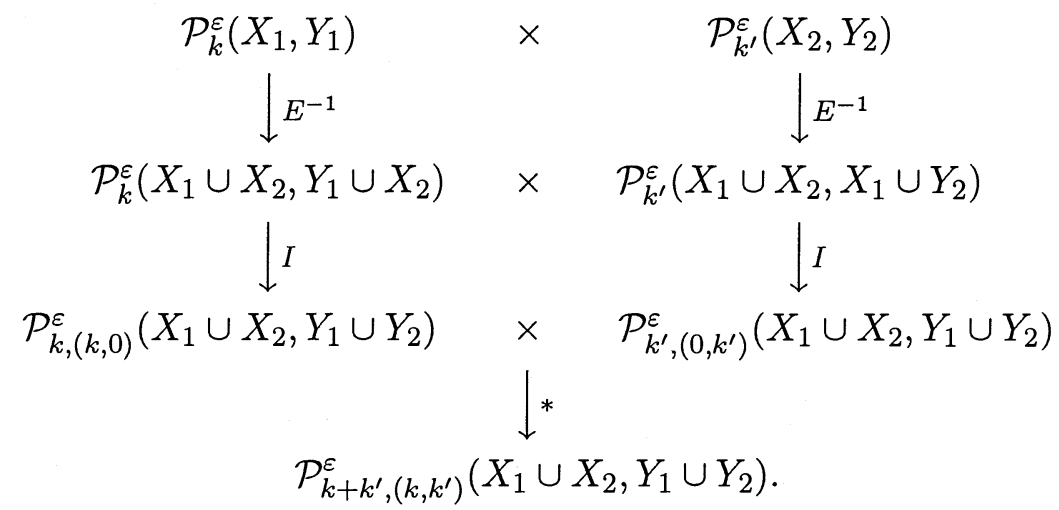

is an isomorphism.

These last two properties (5.12), (5.13) allow us, by decomposition into products, to restrict ourselves, as in the case of $\mathcal{B}(X, Y)$ to considering only $\mathcal{P}_{k}(X, Y)$ for spaces for which $X \backslash Y$ is connected. We now do this.

\section{5.b. Stabilisation.}

The properties above allow us to define stabilisation maps $\mathcal{P}_{k}^{\varepsilon}(X, Y) \rightarrow$ $\mathcal{P}_{k+1}^{\varepsilon}(X, Y)$, when $\varepsilon$ is sufficiently small. Indeed, we choose a point $p$ lying in the the collar of $Y$, choosing the metric so that $p$ lies sufficiently far from $X \backslash Y_{1}$ for the addition of $p$ not to affect the amalgamation sequence of $\sum a_{j} q_{j} \in S P^{k}\left(X \backslash Y_{1}\right)$ in the ranges involved in the construction of $\mathcal{P}_{k}^{\varepsilon}(X, Y)$. We now pick a fixed element $\alpha$ of $\mathcal{M}_{1}^{c}$. Let $\alpha_{\varepsilon}$ be the corresponding connection on the ball of radius $\varepsilon d(1)$ centred at $p$. We then define, for small $\varepsilon$, and up to homotopy, the map

$$
\begin{aligned}
f: \mathcal{P}_{k}^{\varepsilon}(X, Y) & \rightarrow \mathcal{P}_{k+1}^{\varepsilon}(X, Y) \\
\beta & \longmapsto \beta * \alpha_{\varepsilon, j}
\end{aligned}
$$

These maps, up to homotopy, are independent of $\varepsilon$. We can then define a limit space

$$
\mathcal{P}(X, Y)=\lim _{k \rightarrow \infty} \mathcal{P}_{k}^{\varepsilon}(X, Y) .
$$

The limiting process would force us to take smaller and smaller $\varepsilon$, or connections which are more and more concentrated. Alternately, we can make the collar of infinite width. In understanding these spaces $\mathcal{P}(X, Y)$, it is 
perhaps useful to realise that they come equipped with a "virtual charge". This can be defined along the lines of the total charge of the "Dirac sea", as the number of particles not in the stabilisation slots minus the number of slots which are unoccupied. As the topology of the space precludes moving an infinite number of particles continuously, this charge is constant along the space. Thus, for example, adding a particle does not in a strict sense define a map from $\mathcal{P}(X, Y)$ to itself, but rather to a space which is homeomorphic to it.

We will say that $\mathcal{P}(X, Y)$ defines a homotopy model for $\mathcal{B}(X, Y)$ if the limit of the inclusion maps

$$
\mathcal{P}(X, Y) \longrightarrow \mathcal{B}(X, Y)
$$

is a homotopy equivalence.

Theorem (5.17). $\mathcal{P}(X, Y)$ is a homotopy model for $\mathcal{B}(X, Y)$.

Proof. As in $[\mathrm{McD}]$, the proof will proceed inductively, using a Morse function $f$ on $X$, whose unique minimum will be the base point, such that $Y=f^{-1}\left(\left(c_{n+1}, \infty\right)\right)$. If $c_{0}<c_{1}<\cdots<c_{n}$ are the critical values of $f$, then $f^{-1}\left(\left[c_{0}, t\right]\right)$ is a fixed 4-manifold $X_{i}$ for $t \in\left(c_{i}, c_{i+1}\right)$. For such a $t$, choose a $\delta$ with $c_{i}<t-\delta$, and let $\hat{\partial} X_{i}=f^{-1}(t-\delta, t)$. The manifold $\hat{\partial} X_{i}$ is a "thickened boundary" of $X_{i}$. We note that $X_{0} \simeq D^{4}$, the four-ball, and $\hat{\partial} X_{0} \simeq S^{3} \times D^{1}$. If the critical value $c_{i}$ has index $\nu_{i}$, one has:

$$
X_{i} \simeq X_{i-1} \bigcup_{S^{\nu_{i}-1} \times D^{4-\nu_{i}}} D^{4}
$$

One can suppose that $\nu_{i} \in\{1,2,3\}$, for $i=1, \ldots, n, \nu_{0}=0$, and $\nu_{n+1}=4$. For our construction, we are interested in manifolds with "thickened boundary", or a collar. Let $\hat{\partial} X$ denote such a collar for any $X$. The "thickened" version of (5.18) is then:

$$
X_{i} \simeq X_{i-1} \bigcup_{S^{\nu_{i}-1} \times D^{1} \times D^{4-\nu_{i}}} D^{4} .
$$

Now write $D^{4}$ as $D^{\nu_{i}} \times D^{4-\nu_{i}}$, so that

$$
\hat{\partial} D^{4}=\left(S^{\nu_{i}-1} \times D^{1} \times D^{4-\nu_{i}}\right) \bigcup_{S^{\nu_{i}-1} \times D^{1} \times S^{3-\nu_{i}} \times D^{1}}\left(D^{\nu_{i}} \times S^{3-\nu_{i}} \times D^{1}\right)
$$

One has

$$
\hat{\partial} X_{i}=\left(\hat{\partial} X_{i-1} \cup \hat{\partial} D^{4}\right) \backslash V
$$


where

$$
V=\overline{S^{\nu_{i}-1} \times D^{1} \times D^{4-\nu_{i}}}
$$

We have from proposition (2.12) that $\mathcal{B}\left(X_{i-1}, \hat{\partial} X_{i-1}\right)$ is equivalent to $\mathcal{B}\left(X_{i}, \hat{\partial} X_{i} \cup D^{4}\right)$, and from (2.8) that there is a fibration

$$
\operatorname{Map}\left(\left(D^{4}, D^{4} \cap \hat{\partial} X_{i}\right),(G, e)\right) \rightarrow \mathcal{B}\left(X_{i}, \hat{\partial} X_{i} \cup D^{4}\right) \rightarrow \mathcal{B}\left(X_{i}, \hat{\partial} X_{i}\right) .
$$

(In this case, $\operatorname{Map}\left(\left(D^{4}, D^{4} \cap \hat{\partial} X_{i}\right),(G, e)\right)=\operatorname{Map}_{X}\left(\left(D^{4}, D^{4} \cap \hat{\partial} X_{i}\right),(G, e)\right)$ ). Since the space

$$
\operatorname{Map}\left(\left(D^{4}, D^{4} \cap \hat{\partial} X_{i}\right),(G, e)\right) \simeq \Omega^{4-\nu_{i}}(G),
$$

we can rewrite this as a fibration

$$
\mathcal{B}\left(X_{i-1}, \hat{\partial} X_{i-1}\right) \rightarrow \mathcal{B}\left(X_{i}, \hat{\partial} X_{i}\right) \rightarrow \Omega^{3-\nu_{i}}(G) .
$$

We would like a similar picture for the particle spaces. Our particle spaces $\mathcal{P}(X, Y)$ can be thought of as spaces with "repelling boundary", that is, the particles are kept away from the boundary. In the spirit of $[\mathrm{S}]$, we will need another type of particle spaces $Q_{j}, j=1,2,3$, for which at least part of the boundary is "absorbing". Write $D^{4}$ as a product $D^{j} \times D^{4-j}$, and let the thickened boundary of $D^{4}$ be written as

$$
\hat{\partial} D^{4}=\left(S^{j-1} \times D^{1} \times D^{4-j}\right) \cup\left(D^{j} \times S^{3-j} \times D^{1}\right) .
$$

We will think of the first part

$$
V_{1}=\left(S^{j-1} \times D^{1} \times D^{4-j}\right) \backslash\left[\left(S^{j-1} \times D^{1} \times D^{4-j}\right) \cap\left(D^{j} \times S^{3-j} \times D^{1}\right)\right]
$$

of our boundary as being absorbing, and the second part

$$
V_{2}=\left(D^{j} \times S^{3-j} \times D^{1}\right)
$$

as being repelling. More specifically, dividing the interval $D_{1}$ in three, let us split $V_{1}$ into an outer piece $V_{1, o}$, a middle piece $V_{1, m}$ and an inner piece $\bar{V}_{1, i}$ and consider the particle spaces $\mathcal{P}_{k}^{\varepsilon}\left(D^{4}, V_{1, o} \cup V_{2}\right)$. Now we identify these spaces amongst themselves by allowing ourselves to remove any particle whose centre (defined by the map $L$ ) lies in $V_{1, m}$. We suppose that $V_{1, m}$ is sufficiently wide for none of the disks associated to our particles to 
simultaneously intersect $V_{1, o}$ and $V_{1, i}$. Let $Q_{j}$ denote the space resulting from the identifications of the different $\mathcal{P}_{k}^{\varepsilon}\left(D^{4}, V_{1, o} \cup V_{2}\right)$.

As remarked above in section 5.b, adding a particle does not map $\mathcal{P}(X, Y)$ to itself. Rather, if $\mathcal{P}(X, Y)$ has virtual charge zero (set $\mathcal{P}(X, Y)=$ $\left.\mathcal{P}^{0}(X, Y)\right)$ it maps $\mathcal{P}^{0}(X, Y)$ to a space $\mathcal{P}^{1}(X, Y)$ of virtual charge one, which by a diffeomorphism on the base, can be mapped diffeomorphically to $\mathcal{P}^{0}(X, Y)$.

Proposition (5.26). a) Addition of a particle $\mathcal{P}^{0}(X, Y) \rightarrow \mathcal{P}^{1}(X, Y)$ is a homotopy equivalence.

b) There is a quasi-fibration

$$
\mathcal{P}\left(X_{i-1}, \hat{\partial} X_{i-1}\right) \rightarrow \mathcal{P}\left(X_{i}, \hat{\partial} X_{i}\right) \rightarrow Q_{\nu_{i}} .
$$

Recall that a map $r: T \rightarrow S$ is called a quasi-fibration if the inclusion of the fibers of $r$ into the homotopy fiber induces an isomorphism of homotopy groups for all $s \in S$. A criterion for a map to be a quasi-fibration is given by the following lemma (see [DdT, McD]):

Lemma (5.28). Let $S=\bigcup S_{k}$, where each $S_{k}$ is closed and

$$
S_{0} \subset S_{1} \subset S_{2} \subset \cdots \text {. }
$$

Let $r: T \rightarrow S$ be a map. Suppose that for each $k$

1. $r: r^{-1}\left(S_{k}-S_{k-1}\right) \rightarrow\left(S_{k}-S_{k-1}\right)$ is a fibration with fibre $F$, and

2. there is an open subset $W_{k}$ of $S_{k}$ which contains $S_{k-1}$ and there are homotopies $h_{t}: W_{k} \rightarrow W_{k}$, and $H_{t}: r^{-1}\left(W_{k}\right) \rightarrow r^{-1}\left(W_{k}\right)$ such that

a) $h_{0}=i d, h_{t}\left(S_{k-1}\right) \subset S_{k-1}, h_{1}\left(W_{k}\right) \subset S_{k-1}$,

b) $H_{0}=i d$, and $r \circ H_{t}=h_{t} \circ r$,

c) $H_{1}: r^{-1}(x) \rightarrow r^{-1}\left(h_{1}(x)\right)$ is a homotopy equivalence for all $x \in W_{k}$.

Then $r: T \rightarrow S$ is a quasi-fibration with fibre $F$.

We then have a picture of a fibration by strata, with a compatibility condition between the strata. A typical application ([S, McD]) is in studying spaces $C(Y)$ of configurations of particles on a space $Y$, and the effect of restriction to an open subset $V$. Under suitable conditions the restriction 
$C(Y) \rightarrow \tilde{C}(V)$ is a quasi-fibration with fiber $C(Y \backslash V)$ (A piece of the boundary of $V$ must be absorbing; a precise formulation is found in [S, McD]). The strata $S_{k}$ are the sets of configurations in $V$ of at most $k$ particles, and the problem essentially in passing from $S_{k}$ to $S_{k-1}$ is that a particle "leaks out " of the base of the fibration and moves into the fiber $C(Y \backslash V)$. What then makes the map into a quasi- fibration is when addition of a particle in the fibre is a homotopy equivalence. This case is treated extensively in $[\mathrm{S}, \mathrm{McD}$, Gra, Gu].

Our case follows this model quite closely: the $S_{k}$ will be the images $Q_{\nu_{i}, \leq k}$ of $\mathcal{P}_{k}^{\varepsilon}\left(D^{4}, V_{1, o} \cup V_{2}\right)$ in $Q_{\nu_{i}}$. The open subset $W_{k}$ will be the set of configurations in $\mathcal{P}_{k}^{\varepsilon}\left(D^{4}, V_{1, o} \cup V_{2}\right)$ with particles lying in the collar $V_{1, i}$. The homotopy $h_{t}$ simply pushes the particles in $V_{1, i}$ into $V_{1, m} ; H_{t}$ does the same thing in $X_{i}$, just moving particles in the collar. The fact that $H_{1}$ is a homotopy equivalence is the key element in the proof of $b$ ). We proceed inductively in $i$, for both a) and b). First, for $i=0,\left(X_{0}=D^{4}\right)$, since the map $\mathcal{P}^{0}\left(D^{4}, \hat{\partial} D^{4}\right) \rightarrow \mathcal{P}^{1}\left(D^{4}, \hat{\partial} D^{4}\right)$ is just the limit of the stabilisation maps $f_{k}: \mathcal{P}_{k}\left(D^{4}, \hat{\partial} D^{4}\right) \rightarrow \mathcal{P}_{k+1}\left(D^{4}, \hat{\partial} D^{4}\right)$, part a) follows from the proof of the Atiyah- Jones conjecture for $S^{4}$, as it is shown that the stabilisation maps induce homotopy equivalences through an ever increasing range of dimensions. In turn, for $i=1$, this proves part (b), as the map $H_{1}$ on $r^{-1}(t)$ is simply addition of a certain number of particles (which as in [Gr], can be split into a certain number of charge one particles). More generally, part b) for $i$ follows from part a) for $i-1$ in the same way. In turn part a) for $i$ follows from part b) for $i$ using the long exact sequence in homotopy applied to the diagram

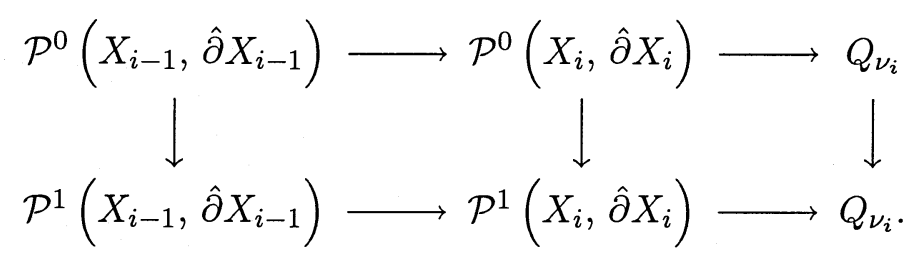

A special case of this quasi-fibration is obtained by splitting the interval $D^{1}$ into three subintervals, and considering the corresponding splitting of $D^{4}=D^{1} \times D^{3}$ into three pieces, with the middle piece serving to define $Q_{1}$. The quasi-fibration (5.27) then becomes:

$$
\mathbb{Z} \times \mathcal{P}\left(D^{4}, \hat{\partial} D^{4}\right) \times \mathcal{P}\left(D^{4}, \hat{\partial} D^{4}\right) \rightarrow \mathcal{P}\left(D^{4}, \hat{\partial} D^{4}\right) \rightarrow Q_{1}
$$


The $\mathbb{Z}$ factor arises because one has two outer components in which particles can live, giving a decomposition of the fiber as

$$
\bigsqcup_{i} \mathcal{P}^{i}\left(D^{4}, \hat{\partial} D^{4}\right) \times \mathcal{P}^{-i}\left(D^{4}, \hat{\partial} D^{4}\right)=\mathbb{Z} \times \mathcal{P}\left(D^{4}, \hat{\partial} D^{4}\right) \times \mathcal{P}\left(D^{4}, \hat{\partial} D^{4}\right) .
$$

One has a diagram

$$
\begin{gathered}
\mathbb{Z} \times \mathcal{P}\left(D^{4}, \hat{\partial} D^{4}\right) \times \mathcal{P}\left(D^{4}, \hat{\partial} D^{4}\right) \longrightarrow \mathcal{P}\left(D^{4}, \hat{\partial} D^{4}\right) \longrightarrow \\
\downarrow \\
\mathbb{Z} \times \mathcal{B}\left(D^{4}, \hat{\partial} D^{4}\right) \times \mathcal{B}\left(D^{4}, \hat{\partial} D^{4}\right) \longrightarrow \mathcal{B}\left(D^{4}, \hat{\partial} D^{4}\right) \longrightarrow \Omega_{1}(G)
\end{gathered}
$$

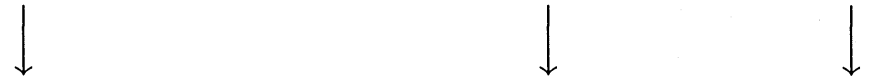

We recall that $\mathcal{B}\left(D^{4}, \hat{\partial} D^{4}\right)=\mathcal{B}\left(S^{4}, p\right)$ is homotopic to a component $\Omega_{0}^{3}(G)$ of $\Omega^{3}(G)$ [AJ]; if the base point $p$ is chosen to be the north pole of $S^{4}$, starting with a connection on $S^{4}$ with a trivialisation at the north pole, we can integrate this connection along a fixed meridian to obtain a trivialisation at the south pole. One then integrates the connection along all meridians starting from the north pole to the equator, and similarily from the south pole to the equator, obtaining two different trivialisations along the equator. Comparing these two trivialisations gives a map from the equator into $G$, that is an element of $\Omega^{3}(G)$, whose degree is essentially the Pontrjagin class of the bundle. The map from $Q^{1}$ to $\Omega^{2}(G)$ is defined in a similar fashion; one just integrates the connections along the $\left(D^{3}, \hat{\partial} D^{3}\right)$ lying in the middle of $\left(D^{4}, \hat{\partial} D^{4}\right)$. The map from $\mathcal{B}\left(D^{4}, \hat{\partial} D^{4}\right)=\mathcal{B}\left(S^{4}, p\right) \simeq \Omega^{3}(G)$ to $\Omega^{2}(G)$ is just restriction to the equator.

The first two downwards arrows in (5.30) are weak homotopy equivalences, by (4.3) and (5.7); from the long exact sequence in homotopy, the map $Q_{1} \rightarrow \Omega^{2}(G)$ is also a weak homotopy equivalence. More generally, one has:

Proposition (5.31). $Q_{i}$ is weakly equivalent to $\Omega^{3-i}(G)$, for $i=1,2,3$.

Proof. Generalising (5.29), we have a quasi-fibration.

$$
Q_{i} \times Q_{i} \rightarrow Q_{i} \rightarrow Q_{i+1}
$$

from which the result follows. 
We can now return to proving theorem (5.17). From (5.24), (5.26), (5.31), we have a diagram

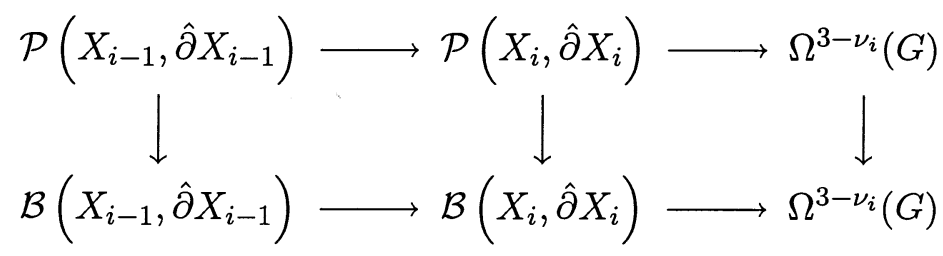

with the horizontals quasi-fibrations and the left-most and right-most downward maps weak homotopy equivalences. The middle downward map is then also a weak homotopy equivalence, completing our inductive step.

\section{Stability of homotopy groups through a range.}

We now have that the spaces $\mathcal{P}(X, Y)=\lim _{k} \mathcal{P}_{k}(X, Y)$ give us a homotopy model for $\mathcal{B}(X, Y)=\mathcal{B}_{k}(X, Y)$. We could ask for the stronger result that the $\mathcal{P}_{k}(X, Y)$ have the same homotopy type as $\mathcal{B}(X, Y)$ through a range, so that $\pi_{i}\left(\mathcal{P}_{k}(X, Y)\right)=\pi_{i}(\mathcal{B}(X, Y))$ for $i<i(k)$, with $i(k)$ increasing monotonically in $k$ and $\lim _{k \rightarrow \infty} i(k)=\infty$ ("stabilisation through a range"). This is indeed what happens with the space $\mathcal{P}_{k}\left(D^{4}, \hat{\partial} D^{4}\right)$, with $i(k)=[k / 2]-2$. To prove this for a general pair, it suffices to show that the stabilisation map induces $\pi_{i}\left(\mathcal{P}_{k}(X, Y)\right)=\pi_{i}\left(\mathcal{P}_{k+1}(X, Y)\right)$ for $i<i(k)$. We would like to proceed as in section 5 , inductively by adding handles, and in particular, we would like the finite particle analogue of (5.25)

$$
F \rightarrow \mathcal{P}_{k}\left(X_{i}, \hat{\partial} X_{i}\right) \rightarrow Q_{\nu_{i}, \leq k}
$$

to behave like a fibration through a range. This is not the case: over an element in $Q_{\nu_{i}, \leq k}$ with total charge $j \leq k$, the fiber $F$ is $\mathcal{P}_{k-j}\left(X_{i-1}, \hat{\partial} X_{i-1}\right)$, and so the fiber varies from a point $(j=k)$ to the full $\mathcal{P}_{k}\left(X_{i-1}, \hat{\partial} X_{i-1}\right)$.

Nevertheless, given a reasonable assumption about the Taubes retraction, that the Taubes retraction commutes up to homotopy with addition of particles, we can show that stabilisation through a range does indeed hold, and we now proceed to sketch how this could be done.

In our induction, we first show that there are subspaces

$$
\tilde{\mathcal{P}}_{k}\left(X_{i}, \hat{\partial} X_{i}\right), \tilde{Q}_{\nu_{i}, \leq k} \text { of } \mathcal{P}_{k}\left(X_{i}, \hat{\partial} X_{i}\right), Q_{\nu_{i}, \leq k}
$$

such that through a range of dimensions the map $\mathcal{P}_{k}\left(X_{i}, \hat{\partial} X_{i}\right) \rightarrow Q_{\nu_{i}, \leq k}$ is homotopically equivalent to $\tilde{\mathcal{P}}_{k}\left(X_{i}, \hat{\partial} X_{i}\right) \rightarrow \tilde{Q}_{\nu_{i}, \leq k}$. We then show that, 
again through a range of dimensions, the map $\tilde{\mathcal{P}}_{k}\left(X_{i}, \hat{\partial} X_{i}\right) \rightarrow \tilde{Q}_{\nu_{i}, \leq k}$ behaves sufficiently like a quasifibration for the induction to go through.

To do this, we can exploit a description of the moduli space which uses complex geometry. There is a natural way of associating to an instanton in $\mathcal{M}_{k}\left(S^{4}\right)$ a holomorphic vector bundle on $\mathbb{P}_{1}(\mathbb{C}) \times \mathbb{P}_{1}(\mathbb{C})$. This can be done directly: the connection allows us to define an integrable $\bar{\partial}$ operator on $\mathbb{R}^{4}$, which then extends to a compactification; alternately [DK], one can simply use the monad construction of section 4: the monad allows one to define a bundle $E$ over $\mathbb{P}_{1}(\mathbb{C}) \times \mathbb{P}_{1}(\mathbb{C})$ as the cohomology $E=\operatorname{ker}\left(B\left(z_{1}, z_{2}\right)\right) / \operatorname{Im}\left(A\left(z_{1}, z_{2}\right)\right)$ of the complex, whose central portion is of course not exact:

$$
0 \rightarrow \mathcal{O}(-1,-1)^{\oplus k} \stackrel{A\left(z_{1}, z_{2}\right)}{\longrightarrow} \mathcal{O}^{\oplus 2 k+2} \stackrel{B\left(z_{1}, z_{2}\right)}{\longrightarrow} \mathcal{O}(1,1)^{\oplus k} \rightarrow 0
$$

Here $\mathcal{O}(-1,-1), \mathcal{O}(1,1)$ are standard holomorphic line bundles on $\mathbb{P}_{1}(\mathbb{C}) \times$ $\mathbb{P}_{1}(\mathbb{C}), z_{1}, z_{2}$ are standard coordinates on $\mathbb{P}_{1}(\mathbb{C}) \times \mathbb{P}_{1}(\mathbb{C})$, and, referring to section $4, A\left(z_{1}, z_{2}\right), B\left(z_{1}, z_{2}\right)$ are the matrices:

$$
A\left(z_{1}, z_{2}\right)=\left(\begin{array}{c}
\tau_{1}-z_{1} \\
\tau_{2}-z_{2} \\
\pi
\end{array}\right), \quad B\left(z_{1}, z_{2}\right)=\left(-\tau_{2}+z_{2}, \tau_{1}-z_{1}, \sigma\right) .
$$

The vector bundles which are obtained in this way are trivial on $\mathbb{P}_{1}(\mathbb{C}) \times$ $\{\infty\} \cup\{\infty\} \times \mathbb{P}_{1}(\mathbb{C})$; conversely any bundle on $\mathbb{P}_{1}(\mathbb{C}) \times \mathbb{P}_{1}(\mathbb{C})$ which is trivial on $\mathbb{P}_{1}(\mathbb{C}) \times\{\infty\} \cup\{\infty\} \times \mathbb{P}_{1}(\mathbb{C})$ can be written in such a form. Furthermore, one can show that there is also a natural framing on $E$ over $\mathbb{P}_{1}(\mathbb{C}) \times\{\infty\} \cup$ $\{\infty\} \times \mathbb{P}_{1}(\mathbb{C})$ associated to (6.2), induced by the last two entries of $\mathcal{O}^{\oplus 2 k+2}$. There is a theorem of Donaldson $[\mathrm{D}]$ which states that such bundles, with their framings, are equivalent to instantons:

Theorem (6.4). The moduli space $\mathcal{M}_{k}\left(S^{4}\right)$ is equivalent to the space of pairs

$$
\left[\begin{array}{c}
\text { holomorphic vector bundles } E \text { on } \mathbb{P}_{1}(\mathbb{C}) \times \mathbb{P}_{1}(\mathbb{C}) \text { with } c_{2}(E)=k, \\
\text { framings of } E \text { along } \mathbb{P}_{1}(\mathbb{C}) \times\{\infty\} \cup\{\infty\} \times \mathbb{P}_{1}(\mathbb{C})
\end{array}\right]
$$

As we saw, the approximate location of the instantons in $\mathbb{C}^{2}=\mathbb{R}^{4}$ was given by the eigenvalues of $\tau_{1}, \tau_{2}$. The eigenvalues of $\tau_{1}$ then give the projections of these approximate locations onto $\mathbb{C}=\mathbb{R}^{2}$, and there is a well defined map

$$
\Pi: \mathcal{M}_{k}\left(S^{4}\right) \rightarrow S P^{k}(\mathbb{C})
$$


corresponding to the eigenvalues. This map was extensively studied in $[\mathrm{BHMM}]$, and one has

Theorem (6.6). There exist complex varieties $V_{1}, V_{2}, \ldots$ with $\operatorname{dim}\left(V_{i}\right)=$ $3 i$ such that the fiber of $\Pi$ over a point $\sum_{i=1}^{j} m_{i} x_{i}, m_{i} \in \mathbb{N}, x_{i} \in \mathbb{C}, x_{i} \neq x_{j}$ is the product $V_{m_{1}} \times V_{m_{2}} \times \cdots \times V_{m_{j}}$. If $\mathcal{S}_{m_{1}, \ldots, m_{j}}$ is the stratum of $S P^{k}\left(\mathbb{C}^{\prime}\right)$ corresponding to a fixed pattern of multiplicities, then $\Pi^{-1}\left(\mathcal{S}_{m_{1}, \ldots, m_{j}}\right)$ is of complex codimension $\sum\left(m_{i}-1\right)$. In fact, $\Pi^{-1}\left(\mathcal{S}_{m_{1}, \ldots, m_{j}}\right)$ is the space:

$$
\begin{aligned}
\left\{\left(x_{1}, \ldots, x_{j}, E_{1}, \ldots, E_{j}\right) \in \mathbb{C}^{j} \times V_{m_{1}} \times\right. & \cdots \times V_{m_{j}} \mid \\
x_{i} & \left.\neq x_{j} \text { if } i \neq j\right\} / \text { Sym },
\end{aligned}
$$

where Sym denotes the subgroup of the symmetric group on $j$ letters which stabilises $\left(m_{1}, \ldots, m_{j}\right)$.

This last statement tells us that $\Pi^{-1}\left(\mathcal{S}_{m_{1}, \ldots, m_{j}}\right)$ is a space of labelled points with multiplicity, with points of multiplicity $m$ labelled by elements of $V_{m}$, and each point labelled independently. With this description, it is natural to hope that homotopies of $S P^{k}(\mathbb{C})$ which preserve the multiplicity stratification should lift naturally to $\mathcal{M}^{k}$. This is indeed the case, at least for the simple homotopy we will consider.

Now let us return to our construction of the manifold $X$ by adding handles, with

$$
X_{i} \simeq X_{i-1} \bigcup_{S^{\nu_{i}-1} \times D^{4-\nu_{i}}} D^{4}
$$

If we are considering the particle space $\mathcal{P}_{k}\left(X_{i}, \hat{\partial} X_{i}\right)$, we can again consider the restriction map $\mathcal{P}_{k}\left(X_{i}, \hat{\partial} X_{i}\right) \rightarrow Q_{\nu_{i}}$. The image of this is defined by the obvious constraint that there can be at most $k$ particles in the handle, so that the target space for restriction will be a space $Q_{\nu_{i}, \leq k}$, rather than $Q_{\nu_{i}}$. We can identify the handle with $\mathbb{R}^{4}$, and our particles are all of suitably low energy for the Taubes retraction to apply. Given an element of $\mathcal{P}_{k}\left(X_{i}, \hat{\partial} X_{i}\right)$, we can apply the retraction to its restriction to the handle to modify it so that one has an instanton over the handle. As one moves around in $\mathcal{P}_{k}\left(X_{i}, \hat{\partial} X_{i}\right)$, particles will move in and out of the handle and this is where one needs an assumption about the Taubes retraction, that, up to homotopy, it should commute in a suitable way with loop sum. This should then allow us to deform the spaces $\mathcal{P}_{k}\left(X_{i}, \hat{\partial} X_{i}\right), Q_{\nu_{i}, \leq k}$ into spaces $\hat{\mathcal{P}}_{k}\left(X_{i}, \hat{\partial} X_{i}\right), \hat{Q}_{\nu_{i}, \leq k}$ of connections whose restriction to the collar would be instantons.

Now let us consider the subspace $\mathcal{M}_{k, j}$ of $\mathcal{M}_{k}$ consisting of those elements whose image under $\Pi$ has at most $j$ points along the imaginary axis, counting 
multiplicity. The complement of this space has real codimension $j$ in $\mathcal{M}_{k}$, and so $\pi_{i}\left(\mathcal{M}_{k}\right)=\pi_{i}\left(\mathcal{M}_{k, j}\right)$ for $i<j-1$. One then should have similarily that the corresponding subspaces $\hat{\mathcal{P}}_{k, j}\left(X_{i}, \hat{\partial} X_{i}\right)$ of $\hat{\mathcal{P}}_{k}\left(X_{i}, \hat{\partial} X_{i}\right)$ are also homotopy equivalent to the full spaces through the range $j-2$. On the space $\mathcal{M}_{k, j}$, viewed as a space of labelled particles, there is a natural homotopy which "chases" the particles away from the imaginary axis, by simply rescaling the real coordinate of their location under the map $\Pi$. Transferring this to the spaces $\hat{\mathcal{P}}_{k, j}\left(X_{i}, \hat{\partial} X_{i}\right)$, one can "chase" all but $j$ of the particles from the handle, so as to obtain after this homotopy a map:

$$
\hat{\mathcal{P}}_{k, j}\left(X_{i}, \hat{\partial} X_{i}\right) \rightarrow \hat{Q}_{\nu_{i}, \leq j}
$$

This map behaves like a quasifibration through a range of dimensions, which grows linearly with $(k-j)$. Indeed, the fiber over a point in $\hat{Q}_{\nu_{i}, \leq j}$ with particle number $\ell \leq j$ is the space $\mathcal{P}_{k-\ell}\left(X_{i-1}, \hat{\partial} X_{i-1}\right)(\ell$ particles in the handle and $k-\ell$ without). The homotopy groups of these spaces for $\ell \leq j$ are isomorphic, by the inductive hypothesis, through a range which grows linearly in $k-j$, and the isomorphisms can be induced by the maps between the spaces $\mathcal{P}_{k-\ell}\left(X_{i-1}, \hat{\partial} X_{i-1}\right)$ and $\mathcal{P}_{k-\ell^{\prime}}\left(X_{i-1}, \hat{\partial} X_{i-1}\right)$ given as in 5.b, by adding fixed particles. This allows us by the corresponding lemma to lemma (5.26) to establish that one does have a quasi-fibration through a range of dimensions. Similarily, as in the previous section, we show that the homotopy groups of $\hat{Q}_{\nu_{i}, \leq j}$ stabilise through a range of dimensions which increases linearly with $j$. Taking $j=[k / 2]$, and considering the long exact sequence of the quasi-fibration shows that the homotopy groups of $\hat{\mathcal{P}}_{k, j}\left(X_{i}, \hat{\partial} X_{i}\right)$, and hence those of $\mathcal{P}_{k}\left(X_{i}, \hat{\partial} X_{i}\right)$ stabilise through a range growing linearly with $k$.

\section{Comments.}

As explained above, the particle model allows natural definitions of homology "operations" on $\mathcal{B}(X, Y)$. When $(X, Y)=\left(S^{4}, D^{4}\right)$, this idea is already present in the work of Boyer and Mann [BoMa], who exploit the isomorphism between the standard "little cubes" structure on $\mathcal{B}\left(S^{4}, D^{4}\right)=$ $\Omega^{4}(B G)$ and the natural one suggested by this particle picture. More generally, the inclusion of $\mathbb{R}^{4}$ into $X \backslash Y$ induces a map $\mathcal{B}\left(S^{4}, D^{4}\right) \rightarrow \mathcal{B}(X, Y)$, which on the level of connections, is simply pull-back from $\left(S^{4}, D^{4}\right)$ to $\left(X, X \backslash \mathbb{R}^{4}\right)$, followed by restriction of the trivialisations from $X \backslash \mathbb{R}^{4}$ to $Y$. This tells us that all the $\mathbb{R}^{4}$ operations carry over naturally to $(X, Y)$.

More generally, choosing a trivialisation of the tangent bundle along a $d$-dimensional cycle in $X$ allows us to transport a configuration of particles in $\mathbb{R}^{4}$ along the cycle, giving us a map

$$
H_{*}\left(\mathcal{B}\left(S^{4}, D^{4}\right), \mathbb{Z}\right) \rightarrow H_{*+d}(\mathcal{B}(X, Y), \mathbb{Z})
$$


It is also worth mentioning that such a particle picture appears in other contexts. One natural case is that of (based) maps of a Riemann surface into $\mathbb{P}^{n}(\mathbb{C})[S]$ (more generally a flag manifold [BHMM2], or even more generally, a suitable class of "almost homogeneous spaces" [BHM]), where the holomorphic maps fulfill the role played here by instantons. Indeed, one can represent the map as a configuration of poles on the Riemann surface, with a principal part attached to each pole: these play the role of particles. The full mapping space then appears as an infinite particle limit of the holomorphic maps. For connections, $S^{4}$ gave us a basic model, to build the space for arbitrary $X$; for maps the model Riemann surface is the twosphere, and the data associated to the two-sphere then gives the appropriate particles for an arbitrary Riemann surface; in this case things are somewhat simpler as the spaces are all locally isomorphic $[\mathrm{Hu}]$.

More generally such particle-like phenomena are often seen to be associated to variational problems of critical exponent, with solutions concentrating around points ("bubbling"). In some sense the purpose of this article is to show that the topology follows the analysis, or vice-versa. For example, one could ask about connections on manifolds of higher dimension, and whether a model of the type we have given should exist. This does not seem plausible. Indeed, to give a connection on an $n$-manifold with curvature concentrated near points, one must be able to trivialise the bundle over the $(n-1)$-skeleton. For $n=4$, this is possible, but for higher dimensions it is not. From an analytical point of view, this seems to be reflected in the fact that in higher dimensions the Yang-Mills functional is no longer of critical exponent. 


\section{References.}

[AJ] M.F.Atiyah and J.D.Jones, Topological aspects of Yang-Mills theory, Comm. Math. Phys., 61 (1978), 97-118.

[ADHM] M.F.Atiyah, V.G.Drinfeld, N.J.Hitchin and Y.I.Manin, Construction of instantons, Phys. Lett. A, 65 (1978), 185-187.

[BHM] C.P. Boyer, J. Hurtubise, and R.J. Milgram, Stability theorems for spaces of rational curves, preprint, $30 \mathrm{p}$.

[BHMM] C.P.Boyer, J.Hurtubise, B.M.Mann and R.J.Milgram, The topology of instanton moduli spaces. I: The Atiyah-Jones conjecture, Ann. of Math. 137 (1993), 561-609.

[BHMM2] C.P.Boyer, J.C.Hurtubise, B.M.Mann and R.J.Milgram, The topology of the space of rational maps into generalised flag manifolds, Acta Math., 173 (1994), 61-101.

[BoMa] C.P.Boyer and B.M. Mann, Homology operations on instantons, J. Diff. Geom. 28 (1988), 423-465.

[CLM] F.R.Cohen, T.J.Lada and J.P. May, The Topology of Iterated Loop Spaces, Springer Lecture Notes 533, Berlin, 1976.

[D] S.K.Donaldson, Instantons and geometric invariant theory, Comm. Math. Phys. 93 (1984), 453-461.

[DdT] A.Dold and R.Thom, Quasifaserungen und unendliche symmetrische Produkte, Ann. of Math. 67 (1958), 239-281.

[DK] S.K.Donaldson and P.B. Kronheimer, The geometry of four-manifolds, Oxford University Press, Oxford, 1990.

[Gra] J.Gravesen, On the topology of spaces of holomorphic maps, Acta Math. 162 (1989), 247-286.

[Gu] M.A.Guest, The topology of the space of rational curves on a toric variety, Acta Math., 174 (1995), 119-145.

[Hu] J.C.Hurtubise, Holomorphic maps of a Riemann surface into a flag manifold, J. Diff. Geom. 43 (1996) 99-118.

[HuMi] J.C.Hurtubise and R.J.Milgram, The Atiyah-Jones conjecture for ruled surfaces J. fur die Reine und Ang. Math. 466 (1995), 111-143.

[J] I.M.James, Reduced product spaces, Ann. of Math. 62 (1955), 170-197. 
[Ka] H.Karcher, Riemannian center of mass and mollifier smoothing, Comm. Pure Appl. Math. 30 (1977), 509-541.

[Ki] F.C.Kirwan, Geometric invariant theory and the Atiyah-Jones conjecture, Sophus Lie Memorial Conference Proceedings, O.A. Laudal et B. Jahren eds. Scandinavian University Press, Oslo, 1994.

[KM] D.Kotschick and J.Morgan, SO(3)-invariants for 4-manifolds with $b_{2}^{+}=1$, J. Diff. Geom. 39 (1994), 433-456.

[Li] J.Li, The first two Betti numbers of the moduli spaces of vector bundles on surfaces, Comm. Anal. Geom. 5 (1997), 625-684.

[May] J.P.May, The Geometry of Iterated Loop Spaces, Springer-Verlag L.N.M. 271 (1972).

[McD] D.McDuff, Configuration spaces of positive and negative particles, Topology, 14 (1975), 91-107.

[Mi] R.J.Milgram, Iterated loop spaces, Ann. of Math. 84(1966) 386-403.

[S] G.Segal, The topology of rational functions, Acta Math. 143 (1979), 39-72.

[T1] C.H.Taubes, Path-connected Yang-Mills moduli spaces, J. Diff. Geom. 19 (1984), 337-392.

[T2] C.H.Taubes, The stable topology of self-dual moduli spaces, J. Diff. Geom. 29 (1989), 163-230.

[T3] C.H.Taubes, A framework for Morse theory for the Yang-Mills functional, Invent. math. 94 (1988) 327-402.

[T4] C.H.Taubes, private communication.

[Ti1] Y.Tian, The based $S U(n)$-instanton moduli spaces, Math. Ann. 298 (1994) 117-140.

[Ti2] Y.Tian, The Atiyah-Jones conjecture for classical groups and the Bott periodicity, J. Diff. Geom., 44 (1996), 178-199.

[U] K.Uhlenbeck, Removable singularities in Yang-Mills fields, Comm. Math. Phys. 83 (1982), 11-30.

Received November 13, 1997. 
MCGILl UNIVERSITY

805 SHERBRoOKe St.W.

Montreal, Canada H3A $2 \mathrm{~K} 6$

E-mail address: hurtubis@gauss.math.mcgill.ca 